\title{
Quantized transport, strain-induced perfectly conducting modes and valley filtering on shape-optimized graphene Corbino devices ${ }^{\dagger}$
}

\author{
Gareth W. Jones, ${ }^{\ddagger, \perp}$ Dario Andres Bahamon, ${ }^{\Phi, \perp}$ Antonio H. Castro Neto,,,$\|$ and \\ Vitor M. Pereira*,§,\| \\ $\ddagger$ School of Mathematics, The University of Manchester, Manchester, M13 9PL, England \\ \MackGraphe - Graphene and Nano-Materials Research Center, Mackenzie Presbyterian University, Rua da \\ Consolação 896, 01302-907, São Paulo, SP, Brazil \\ $\S$ Department of Physics, National University of Singapore, 2 Science Drive 3, Singapore 117542 \\ \|Centre for Advanced 2D Materials, National University of Singapore, 6 Science Drive 2, Singapore 117546 \\ $\perp$ These authors contributed equally to this work. \\ E-mail: vpereira@nus.edu.sg \\ Phone: +65 66013642
}

\begin{abstract}
The extreme mechanical resilience of graphene ${ }^{112}$ and the peculiar coupling it hosts between lattice and electronic degrees of freedom ${ }^{3-5}$ have spawned a strong impetus towards strain-engineered graphene where, on the one hand, strain augments the richness of its phenomenology and makes possible new concepts for electronic devices $\frac{677}{}$ and, on the other hand, new and extreme physics might take place $\frac{8}{15}$ Here, we demonstrate that the shape of substrates supporting graphene sheets can be optimized for approachable experiments where strain-induced pseudomagnetic fields (PMF) can be tailored by pressure for directionally selective electronic transmission and pinching-off of current flow down to the quantum channel limit. The Corbino-type layout explored here furthermore allows filtering of charge carriers according to valley and current direction, which can be used to inject or collect valley-polarized currents, thus realizing one of the basic elements required for valleytronics. Our results are based on a framework developed to realistically determine the combination of strain, external parameters, and geometry optimally compatible with the target spatial profile of a desired physical property - the PMF in this case. Characteristic conductance profiles are analyzed through quantum transport calculations on large graphene devices having the optimal shape.
\end{abstract}

\footnotetext{
${ }^{\dagger}$ This work has been published in Nano Letters on 4 August 2017, with the DOI 10.1021/acs.nanolett.7b01663.
}

\section{Introduction}

An extremely active and promising area of research in strainengineered two-dimensional electronic systems is the realization of graphene-based nanostructures with tailored pseudomagnetic fields (PMF). The PMF is a fruitful concept in strained graphene arising from the fact that, in the vicinity of the Dirac point, the description of the coupling between lattice deformations and electrons can be captured by two types of strain-induced fields: a displacement potential that couples to electrons as an electrostatic potential, and a strain-induced gauge field that couples similarly to a magnetic field. ${ }^{4}$ Since the former are expected to be effectively screened by the charge carriers, electrons are then expected to respond to strain mostly through this pseudomagnetic effect. Since (pseudo)magnetic fields are very effective means of guiding the motion and confining charged carriers, this naturally led to proposals and studies of many analogues of magnetic devices using graphene without actual magnetic fields, such as pseudomagnetic barriers $\frac{6 \sqrt{66 \mid 18}}{18}$ or pseudomagnetic quantum dots. $\frac{8119}{}$

There are significant advantages of using pseudomagnetic fields in graphene electronic devices at the nanoscale. The first is that electrons in graphene are not easily confined by electric fields due to the Klein tunneling effect ${ }^{20}$ arising from the relativistic-like behavior of electrons in this system. This has been a perennial difficulty and disadvantage of graphenebased electronics, while PMFs can efficiently localize electrons or act as tunneling barriers. ${ }^{6}$ The second advantage is that PMFs are only felt by the electrons in graphene, and they can be confined to regions as narrow as $5-10 \mathrm{~nm}$. 1221 This is unlike real magnetic fields which are felt not only by the target electronic system but also by whole the environment nearby. In addition, fabricating real magnetic barriers of nanoscale size is practically impossible and their mag- 
netic field would also be fixed in principle, not reconfigurable or tuneable. Finally, the magnitude of PMFs that can be achieved in graphene is remarkably high and in excess of $300 \mathrm{~T}^{12 \sqrt{13 / 21}}$ which means that values of the order of tens of Tesla are easily obtainable, and strong enough for many electronic applications.

There is a key challenge that has persisted since the early proposals for strain-engineered graphene: the ability to tailor the spatial distribution of the PMF in graphene hinges on the ability to define specific non-uniform strain profiles, which is a difficult experimental prospect, especially at the micro and nanoscale. Moreover, whereas it is a straightforward theoretical task to start from a given strain field and determine the associated PMF and its impact in the electronic and transport properties, it is much less trivial and non-unique to request the opposite. Yet, it is precisely the opposite (i.e., specifying the PMF patterns in real space and obtaining the necessary strain profiles) that is of most direct interest for application in electronic devices because, in nearly any such case, one should be able to specify how, where, and to which extent we need electrons to be confined, which can be easily done reasoning in terms of PMF patterns alone, but is generally hard to anticipate in terms of strain alone. The central theoretical question for actual realizations and applications of strain-engineered graphene then becomes: how should one design the experimentally accessible parameters of the system (shape, external forces, constraints, substrate features, contacts, etc.) so that any given desired PMF pattern can be generated under realistic experimental circumstances?

In this work we pose and address one such question. We demonstrate that the geometry of a substrate can be optimized so that a graphene Corbino-type device can have its conductance suppressed when hydrostatically pressurized. This, on the one hand, can lead to extremely high on/off ratios due to the two contrasting transport regimes in the pressurized and relaxed states. Pressurized devices are shown to leak current only through perfect, spatially separated $1 \mathrm{D}$ modes arising from snake-type states that are stabilized at the inversion regions of the PMF. This creates a very strong current pinch-off by squeezing (literally in this case) the transport channel to the quantum wire limit and, in addition, establishes the possibility of having strictly quantized conductance on demand without electrostatic confinement. Moreover, since the chirality of PMF-induced snake states is uniquely tied to the valley degree of freedom, such devices can function as effective valley filters or sources of valley polarized currents. Our calculations are performed in an optimization framework that tackles the "reverse" strain-engineering problem in graphene. We begin with an overview of its main ingredients and a discussion of the optimal shapes for nearly uniform PMF throughout the Corbino ring. Subsequently, we describe how the deformation fields so obtained are mapped to an actual graphene lattice whose electronic properties are described in a nonuniform tight-binding approximation. This constitutes the basis for our study of the quantum transport characteristics and local electronic structure in devices of different dimensions, with and without disorder, whose results establish the behavior and phenomenology indicated above.

\section{Corbino shape optimization}

Stating the problem as an optimization question, we wish to find the shape of a non-circular annular cavity in a substrate such that the graphene flake, when deposited on it from above and subjected to hydrostatic pressure of magnitude $p$, exhibits a pseudomagnetic field (PMF) with a magnitude $B_{0}$ that is constant in as much area of the system as possible, compatible with realistic elastic constraints. The substrate shape and dimensions so determined would constitute the basic information for the design of the corresponding transport experiment. If we assume that the graphene flake is rigidly attached to the substrate, finding the shape of the cavity is equivalent to finding the shape of the graphene flake with clamped boundary conditions at its edges, with the same optimized PMF under the applied hydrostatic pressure. This problem is solved by appealing to a Partial Differential Equation (PDE) constrained optimization technique, discretized by finite elements. ${ }^{22}$ It involves varying the control variables (the shape of the cavity) and the state variables (the plate deformation) so as to minimize a measure of the distance of the underlying PMF away from a certain desired PMF pattern, subject to the constraint that the state variables must satisfy the equations governing the elastic deformation of the graphene sheet for a given shape.

\section{Overview of the inverse optimization problem}

Our control variable is the shape of the domain occupied by the graphene flake (it is this that we numerically vary in order to approach the desired PMF in the pressurized field). We thus parametrize the domain using a set of control variables: $\Omega=\Omega\left[c_{1}, \ldots, c_{n}\right]$, to be specified later. The clamped boundary conditions at the two perimeters (and beyond) where the graphene sheet contacts the substrate imply that the material domain of interest is the annular region defined by the patterning of the substrate, and not that part of the graphene that adheres to the substrate itself. The central mathematical problem is as follows:

$$
\begin{aligned}
& \text { Minimize } \mathcal{I}=\frac{1}{\operatorname{area} \Omega} \iint_{\Omega\left[c_{1}, \ldots, c_{n}\right]}\left(B^{2}-B_{0}^{2}\right)^{2} \mathrm{~d}^{2} \boldsymbol{X} \\
& +\eta \mathcal{I}^{\text {reg }}\left[c_{1}, \ldots, c_{n}\right] \text {, }
\end{aligned}
$$

subject to six constraint equations expressing the mechanical equilibrium of the graphene sheet, where the PMF $B$ is an explicit and known function of the strain field in the $2 \mathrm{D}$ sheet, and $B_{0}$ is the target PMF which, in general can be any predefined function of space. This formulation ensures that the $B$ sought in the optimization satisfies $B^{2} \approx B_{0}^{2}$ as closely as is feasible under the restrictions caused by the geometry and mechanics of the problem (the scheme seeks PMFs that are as close as possible to $\pm B_{0}$; in the Supporting Information we discuss the case where the objective function is chosen to minimize $B-B_{0}$, rather than $B^{2}-B_{0}^{2}$ ). $\Omega$ represents the domain occupied by the unadhered part of the graphene flake, defined by a characteristic lengthscale $L$. The explicit constraint equations that accompany eq. (1) are provided in the Supporting Information in order not to ob- 
scure the main discussion here. The final term is a regularization term necessary for well-posedness of the optimization problem. In reality, the optimization problem will be solved in dimensionless form but we present the dimensional version here for clarity.

\section{Discretization and numerical solution}

Each of the state variables (the three displacement components $v_{1}, v_{2}, w$ of the plate, and the three components of the bending moment $M_{11}, M_{12}, M_{22}$ ) is discretized using linear finite elements. This requires the generation of a triangular mesh covering the entire domain occupied by the graphene flake. However, the shape of this domain $\Omega$ must be parametrized by a finite set of variables in order for the optimization algorithm (which works in a discrete setting) to be appropriately formulated. The simplest way to parametrize the shape of the domain $\Omega$ is to prescribe equidistant control points around the outer and inner boundaries, and interpolate them with a Fourier series in the angular coordinate $\theta$. Furthermore, due to the symmetry of the graphene lattice, the PMF is invariant if the coordinate axes are rotated by an angle of $2 \pi / 3$. Therefore we may restrict our domain to the region $-\pi / 3 \leq \theta \leq \pi / 3$, and admit outlines of the form

$$
R(\theta)=\sum_{k}\left(\alpha_{k} \cos 3 k \theta+\beta_{k} \sin 3 k \theta\right)
$$

only. An example of the domain construction from control points is shown in Figure 1(a). Let $N$ be the (even) number of control points on the outer boundary, with the same number on the inner boundary. Then if $\left(R_{i}^{\text {out }}\right)_{i=1, \ldots, N}$ are the radial coordinates of the outer boundary for the angular coordinates $\theta_{i}=2 \pi(i-N / 2) /(3 N)$, we have

$$
R_{\text {out }}(\theta)=\frac{\alpha_{0}}{2}+\sum_{k=1}^{N / 2} \alpha_{k} \cos 3 k \theta+\sum_{k=1}^{N / 2-1} \beta_{k} \sin 3 k \theta,
$$

where the coefficients are found by solving $R_{\text {out }}\left(\theta_{i}\right)=R_{i}^{\text {out }}$ for $i=1, \ldots, N$. The same procedure is repeated for the inner boundary control variables $R_{i}^{\text {in }}$. The $2 N$ control variables are allowed to vary within a user-provided interval,

$$
L_{\min }^{\text {in }} \leq R_{i}^{\text {in }} \leq L_{\max }^{\text {in }}, \quad L_{\min }^{\text {out }} \leq R_{i}^{\text {out }} \leq L_{\max }^{\text {out }}
$$

For each set of control variables chosen during the iterative optimization process, we will have a domain of a different shape. It would be improper to triangulate each new domain separately: not only would it be prohibitively timeconsuming, but most optimization routines work best when the constraints and objective functions vary smoothly when the state and control variables are altered. Therefore, we instead choose a mesh for an annular sector and deform the mesh as the control variables accordingly alter the domain. The annular sector is defined by $L_{\text {in }} \leq R \leq L_{\text {out }}$ and $-\pi / 3 \leq \theta \leq \pi / 3$, where

$$
L_{\mathrm{in}}=\left(L_{\min }^{\mathrm{in}}+L_{\max }^{\mathrm{in}}\right) / 2, \text { and } L_{\mathrm{out}}=\left(L_{\min }^{\mathrm{out}}+L_{\mathrm{max}}^{\mathrm{out}}\right) / 2 .
$$

To mesh this annulus in a way which ensures a good resolution at the inner boundary, we construct a uniform mesh of $N_{p}$ points for the rectangle 1

$$
\left\{(x, y): \log L_{\text {in }} \leq x \leq 0, \quad-\pi / 3 \leq y \leq \pi / 3\right\} .
$$

We conformally map the mesh for the rectangular domain to the annular sector using the mapping $X+\mathrm{i} Y=\exp (x+$ iy). Then, to deform this mesh to the domain defined by the control variables, we simply kept the $\theta$-component of the meshpoint fixed, and affinely displaced the radial component $R=\sqrt{X^{2}+Y^{2}}$ to

$$
R_{\text {in }}(\theta)+\left(R_{\text {out }}(\theta)-R_{\text {in }}(\theta)\right)\left(\frac{R-L_{\text {in }}}{L_{\text {out }}-L_{\text {in }}}\right),
$$

where $R_{\text {out }}(\theta)$ and its equivalent at the inner boundary are calculated from the control points by $(3$. See Figures $1(\mathrm{~b})$ 1(d) for a visualization of this procedure. The purpose of the regularization term $\mathcal{I}^{\text {reg }}$ in the objective function (1) is to make the optimization problem well-posed by penalizing solutions that have unfavorable properties. In this study we set the regularization function to be

$$
\mathcal{I}^{\text {reg }}=\int_{-\pi / 3}^{\pi / 3}\left(R_{\text {out }}^{\prime \prime}(\theta)^{2}+R_{\text {in }}^{\prime \prime}(\theta)^{2}\right) \mathrm{d} \theta .
$$

in order to penalize highly-convoluted boundaries.

As discussed previously, the state variables are $v_{1}, v_{2}$, $w, M_{11}, M_{12}, M_{22}$, and are discretized such that the equations are written in terms of their values at each node in the triangulation. Additionally there are boundary conditions to be applied: the displacements are set to zero on nodes comprising the inner and outer boundaries. At the boundaries $\theta= \pm \pi / 3$, we impose rotated periodicity conditions on these quantities (expressed in polar coordinates $R, \theta$ ), i.e. $\left.w\right|_{\theta=\pi / 3}=\left.w\right|_{\theta=-\pi / 3}$, and similarly for $v_{R}, v_{\theta}, M_{R R}$, $M_{R \theta}, M_{\theta \theta}$. The discretized PDE system becomes a discrete optimization system: to minimize an objective function $F(\boldsymbol{C}, \boldsymbol{U})$ subject to $6 N_{p}$ constraints $\boldsymbol{G}(\boldsymbol{C}, \boldsymbol{U})=\mathbf{0}$, where $\boldsymbol{C}$ is the vector of control variables and $\boldsymbol{U}$ is the vector of $6 N_{p}$ state variables.

\section{Optimized device shapes}

As the electronic and mechanical properties of graphene remain fixed, there are only three parameters that we can change in this system: the limits on the annulus shape (and hence the lengthscale $L$ ), the applied (constant) pressure $p$, and the target PMF $B_{0}$. In our calculations we allowed the $N=4$ outer control points to vary in a range $261 \AA \pm 60 \AA$ and the inner points in a range $61 \AA \pm 15 \AA$. We initially set a target PMF of $B_{0}=10 \mathrm{~T}$ and the pressure was fixed at $100 \mathrm{bar}=10^{7} \mathrm{~Pa}$. Writing the coefficients to the nearest $0.1 \AA$, the shape of the optimal outer and inner boundaries

\footnotetext{
${ }^{1} \mathrm{~A}$ mesh size giving around 8 boundary elements per control point was used for the calculations in this article. Meshes were constructed using the Matlab-based mesh routine DistMesh.23
} 


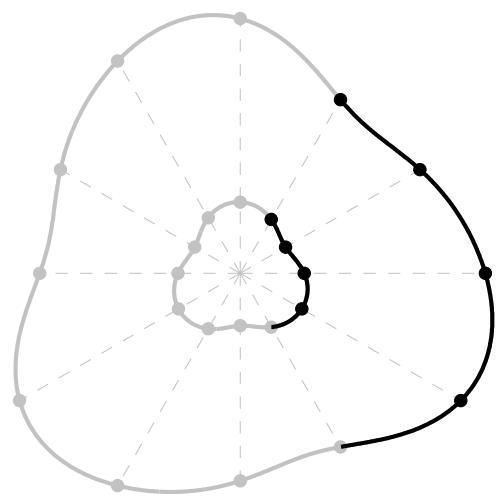

(a)

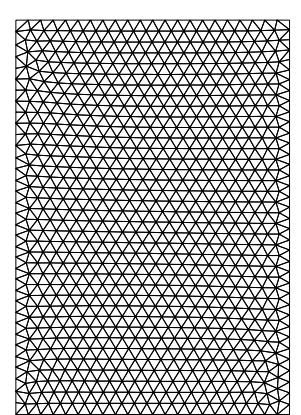

(b)

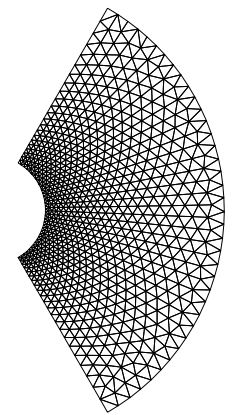

(c)

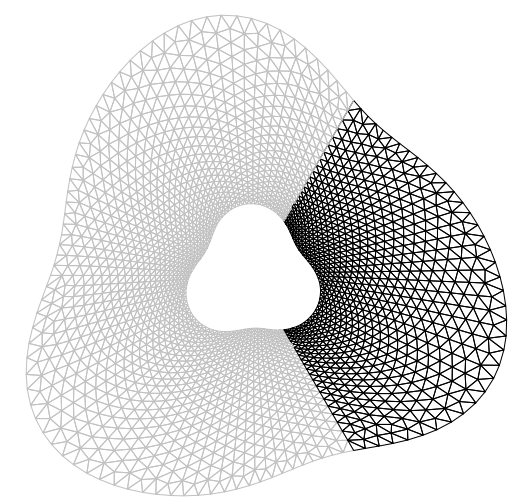

(d)

Figure 1: (a) Construction of the domain shape from a discrete set of $N=4$ control points for the inner and outer boundaries. (b) A uniform grid on a rectangle. The $x$-coordinates of the upper and lower boundaries must be in registration for the periodicity condition to apply. (c) The grid of (b) has been mapped conformally to an annular sector to increase the resolution at the inner boundary. (d) The grid of (c) has been stretched radially to match the example shape shown in (a). This mesh is repeated twice to form a complete mesh, though only the sector in black is calculated.

were calculated to be (in Angstroms)

$$
\begin{gathered}
R_{\text {out }}(\theta)=245.4+0.0 \cos 3 \theta-44.4 \cos 6 \theta+2.0 \sin 3 \theta, \\
R_{\text {in }}(\theta)=47.6+0.1 \cos 3 \theta+0.6 \cos 6 \theta-0.3 \sin 3 \theta
\end{gathered}
$$

This outline corresponds to a striking flower-like geometry, and its deformation under hydrostatic pressure is displayed in Figure 2(a), To this deformation corresponds the spatial distribution of PMF shown in Figure 2(b) Though the target PMF was $10 \mathrm{~T}$, the root-mean-squared value of the calculated PMF was only $4.05 \mathrm{~T}$. It should be kept in mind that the target PMF is not in general attained in these optimization calculations, but is rather a mechanism for forcing the solution towards our desired direction.

Since solutions to the optimization problem in eq. (1) are obtained for a specific choice of input parameters $p$ and $B_{0}$, one immediate and important question is to assess how robust is the solution for the shape with respect to changes in those parameters. As the optimization and its constraints were solved in a dimensionless setting, the behavior of the solution is dependent on the values of dimensionless parameters rather than true physical quantities. The nondimensionalized system has only two parameters: the dimensionless bending stiffness $\kappa$, and the dimensionless pressure $\bar{p}$. In terms of the three physical quantities $p, L$, and $B_{0}$, these parameters behave as (details in the Supporting Information)

$$
\bar{p} \propto \frac{p}{B_{0}^{3 / 2} L^{1 / 2}}, \quad \kappa \propto \frac{1}{L^{3} B_{0}} .
$$

Varying $\kappa$ has little effect on the shape obtained through the optimization procedure (see Fig. S1 and related discussion in the Supporting Information), and so only changes in $\bar{p}$ are relevant in understanding how the optimized shape depends on the choices of input parameters. For instance, increasing the hydrostatic pressure $p$ by a factor of 8 and the target PMF $B_{0}$ by a factor of 4 has no effect on the optimized shape, as the dimensionless constant $\bar{p}$ is kept unchanged and the effect of a changed $\kappa$ is negligible. In contrast, if $\kappa$ is kept constant and $\bar{p}$ is allowed to vary, we see a significant variation in the calculated shape as shown in Figures $3 \mathrm{a}-\mathrm{d}$. This variation in shape with changes in $\bar{p}$ deserves further explanation. A change in $\bar{p}$ corresponds to varying $p$ while keeping the lengthscale and target PMF constant. But a better way of seeing the effect is to think about an increase in $\bar{p}$ as a decrease in target PMF $B_{0}$, while keeping $p$ and $L$ constant [cf. eq. [11]]. Though this also changes the value of $\kappa$, we have noted that the effect of this is minor. To explore this line of thinking, in the lower row of Figure 3 , we report a further set of calculations which kept $p=100$ bar and $L=261 \AA$ fixed while varying the target PMF $B_{0}$ through $50 \mathrm{~T}, 20 \mathrm{~T}, 10 \mathrm{~T}$, and $4 \mathrm{~T}$. For high target PMFs, the optimization forces the inner boundary to be highly convoluted in order to obtain the high strain gradients needed to achieve the target PMF. For low target PMFs, an annular solution is already close to the optimal solution, and the optimization only needs to make small adjustments to the outline to make the magnitude of the field more uniform. The results for $10 \mathrm{~T}$ and $20 \mathrm{~T}$ can be seen as intermediates between these two extremes. The two extremes also explain the results of Figure $3 \mathrm{a}-\mathrm{d}$ calculated using different pressures for a given $B_{0}$ : for a low pressure, a convoluted interior boundary is required to hit the target PMF, while for high pressure an annular solution is already close to the target and only minor modifications of shape are needed. In the end, the two rows of Figure 3 illustrate what is implied by eq. (11): the changes in the optimal shape with increasing $p$ (and fixed $B_{0}$ ) follow the same trend as the changes with decreasing $B_{0}$ (and fixed $p$ ). Of the calculations shown in Figure $3 \mathrm{z}-\mathrm{h}$ we selected the solution $(\mathrm{g})$, which was shown before in Figure 2, with $B_{0}=10 \mathrm{~T}$ and $\bar{p}=0.337$ as giving a good middle ground between PMF smoothness and strength. 


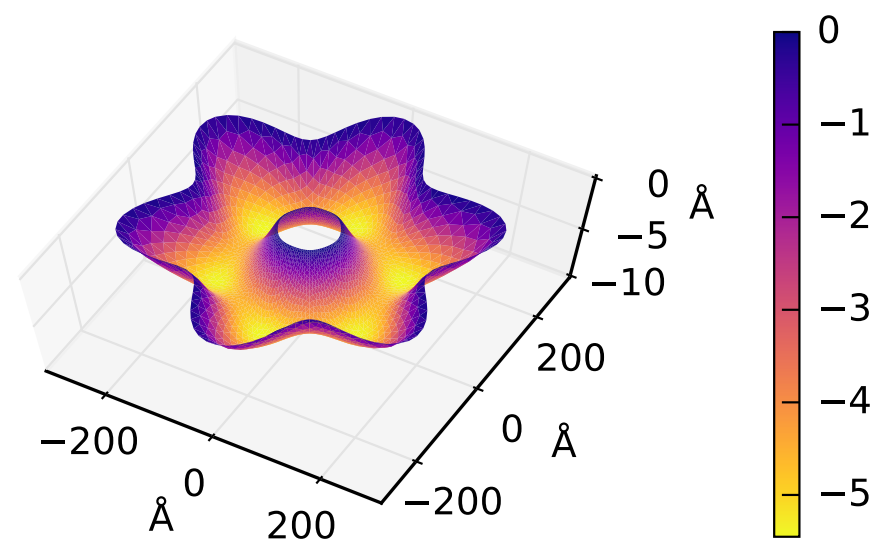

(a)

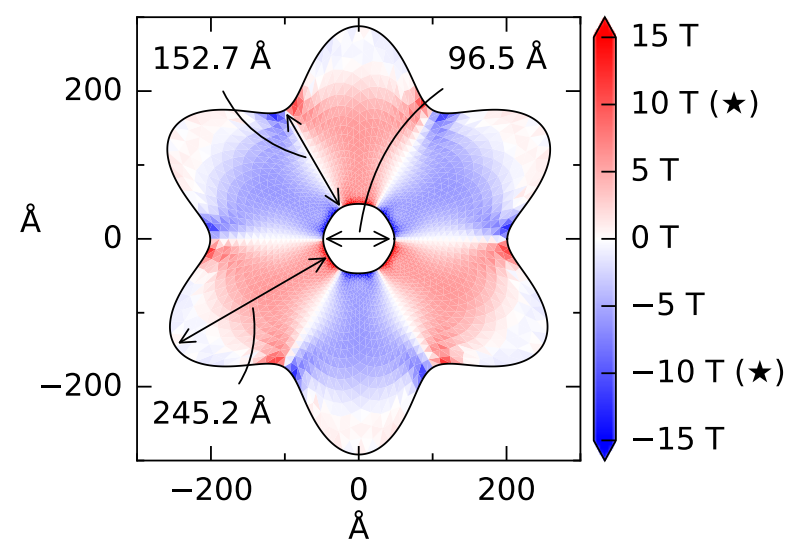

(b)

Figure 2: (color online) (a) An elevation plot (with exaggerated vertical scale) of the pressurized 'flower'-shaped solution. Colors indicate elevation, to a largest value 5.5 $\AA$. (b) The corresponding PMF. Target PMF was $B_{0}=10 \mathrm{~T}$ (indicated by $\star$ on the colorbar). Characteristic lengthscales are indicated on the diagram.

If we are now given an arbitrary lengthscale $L$ and pressure $p$, in order to find the optimal shape we need to choose an appropriate value of $B_{0}$. Since we have seen that the result for $\bar{p}=0.337$ gives an acceptable outcome, we could choose a $B_{0}$ that results in this value of $\bar{p}$. This gives us a system of equations with the same value of $\bar{p}$ but a different value of $\kappa$ as our first calculation. We have noted above that varying $\kappa$ has little effect on the shape of the outline at constant $\bar{p}$. So, as long as the new value of $\kappa$ does not fall too much beyond the range of magnitudes where we verified this to be true (supplementary Fig. S1), the solution will be similar to that in equations (9, 10), plotted in Figure 2(b) Since the differences are small, and would be dwarfed by manufacturing variability in a real system, we kept the same shape $(9)-(10)$ in all our atomistic transport calculations to be discussed below for the sake of comparability. This shape is henceforth referred to as the flower device. The initial optimization was for a pressure of $p=100 \mathrm{bar}$, and we performed further pressurizations of the shape up to $5 \mathrm{kbar}$, for which the distribution of PMF was not greatly changed, though its magnitude was quite different. Each of these pressurizations is identified below by the maximum strain achieved in the graphene sheet, up to a maximum strain of $\varepsilon_{m}=6.11 \%$ (note, however, that, even though our analysis is based on a set of specific parameter values, the scaling relations (11) allow the freedom to explore a large range of relevant pressures, target PMFs, or system sizes without having to run a new shape optimization procedure for each particular choice; see, for example, Fig. S6 in the Supporting Information).

\section{Transport characteristics}

To access the transport properties of the flower device we use the Landauer-Büttiker formalism; specifically, we are interested in the quantum conductance of the device, which is calculated by Caroli's formula ${ }^{24} G=\frac{2 e^{2}}{h} \operatorname{Tr}\left[\Gamma_{q} G^{r} \Gamma_{p} G^{a}\right]$, where $G^{r}=\left[G^{a}\right]^{\dagger}=\left[E+i \eta-H-\Sigma_{p}-\Sigma_{q}\right]^{-1}$ is the retarded [advanced] Green's function, the coupling between the contacts and the device is represented by $\Gamma_{q}=$ $i\left[\Sigma_{q}-\Sigma_{q}^{\dagger}\right]$, and $\Sigma_{q}$ is the self-energy of the contact $q$. The geometry of the device is easily included in a nearest-neighbors $\pi$-band tight-binding Hamiltonian by merely selecting the sites that are located between the mathematically defined inner and outer edges [cf. Figure 2(b)]. The inner contact is essentially a circular contact with $R_{\text {in }} \approx 47 \AA$; the outer contact radius varies between $200 \AA \leq R_{\text {out }} \leq 322 \AA$ with the angular modulation prescribed by eq. (9). The calculation of the conductance requires the Green's functions of these non traditional contacts; this problem can be resolved by recalling that, for graphene, the particular details of the contacts can be replaced by an effective self-energy term provided that the contacts inject a high number of modes (highly doped contacts). ${ }^{[25}$ Under this model, an effective self-energy term $-i|t|$ is added to the onsite energy of the atoms at the edges ( $t=-2.7 \mathrm{eV}$ is the graphene hopping parameter).

The mechanical deformations induced by the hydrostatic pressure are incorporated in the tight binding Hamiltonian through the modification of the hopping parameter between neighboring sites as ${ }^{26}$

$$
\begin{aligned}
t_{i j}= & V_{p p \pi}\left(d_{i j}\right) \widehat{n}_{i} \cdot \widehat{n}_{j} \\
& +\left[V_{p p \sigma}\left(d_{i j}\right)-V_{p p \pi}\left(d_{i j}\right)\right] \frac{\left(\widehat{n}_{i} \cdot \vec{d}_{i j}\right)\left(\widehat{n}_{j} \cdot \vec{d}_{i j}\right)}{d_{i j}^{2}}
\end{aligned}
$$

where $\widehat{n}_{i}$ is the vector normal to the surface at point $i, \vec{d}_{i j}$ is a vector linking the two sites, and $V_{p p \sigma}$ and $V_{p p \pi}$ are the Slater-Koster parameters, modified to account for changes in the bond length through $V_{p p \pi}\left(d_{i j}\right)=t e^{-3.37\left(d_{i j} / a-1\right)}$ and $V_{p p \sigma}\left(d_{i j}\right)=-2.4 V_{p p \pi}\left(d_{i j}\right) .27 .29$

To best appreciate the features that arise from the pressureinduced PMF, it is useful, first, to have a perspective over the main transport characteristics of unpressurized devices which define our scenario of reference; the results of these calculations are provided in the Supporting Information. Devices were generated from the optimized flower shape shown 

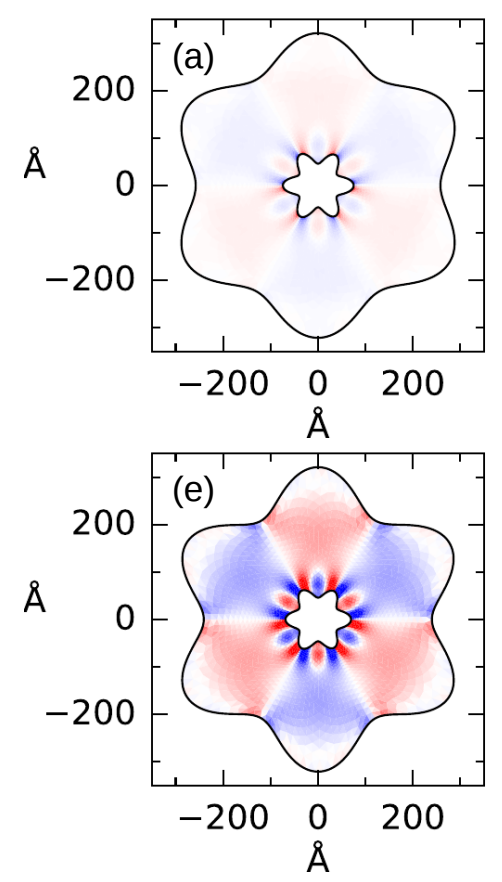
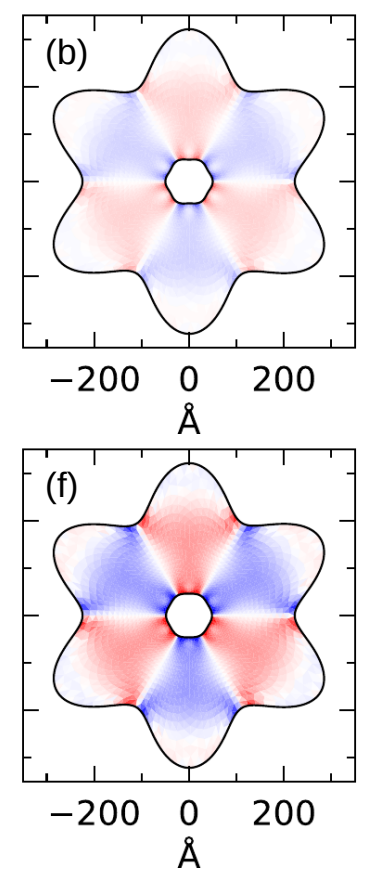
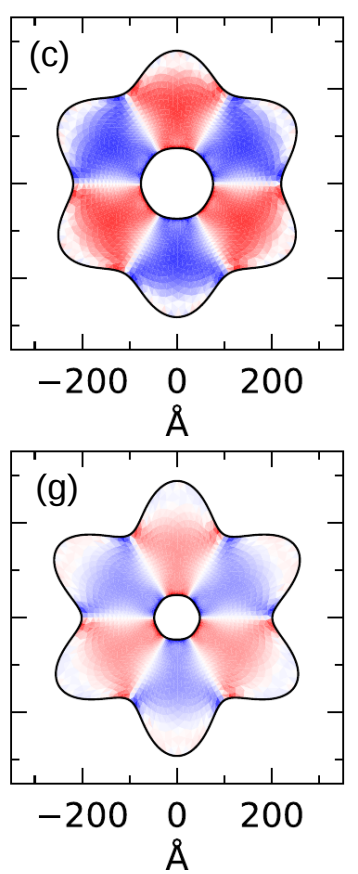
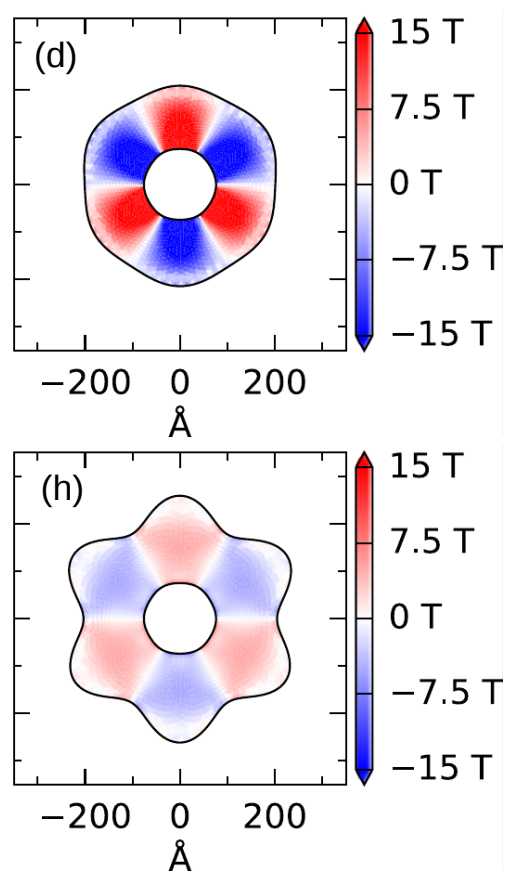

Figure 3: (color online) Upper row (a-d): comparison of the shape and PMF distribution of devices optimized for a target $B_{0}=10 \mathrm{~T}$ and fixed $\kappa=5.76 \times 10^{-5}$. Each panel is the result of the optimization at a different pressure: $p \in\{10 \mathrm{bar}, 10 \sqrt{10} \mathrm{bar}, 100 \sqrt{10} \mathrm{bar}, 1 \mathrm{kbar}\}$ (this corresponds to the set of dimensionless pressures $\bar{p} \in\{0.0337,0.107,1.07,3.37\})$. Lower row (e-h): result of the optimization for different target PMFs of $50 \mathrm{~T}, 20 \mathrm{~T}, 10 \mathrm{~T}$, and 4 T. Pressure was kept fixed at 100 bar and $L=261 \AA$. In all panels (a-h) we used $L=261 \AA$.

in Figure $2(\mathrm{~b})\left(R_{\text {in }} \approx 47 \AA\right.$ and $\left.200 \AA \leq R_{\text {out }} \leq 322 \AA\right)$. Figure 4 a shows the conductance of pressurized flower devices for different values of the maximal strain in the graphene flake, $\varepsilon_{\mathrm{m}}$, that we use to distinguish different cases. Notably, at $\varepsilon_{\mathrm{m}} \approx 6 \%$, the conductance starts developing a plateau of $5.7\left(2 e^{2} / h\right)$ with a resonant peak at $E=0.09 t$; the plateau occupies the whole energy range $0 \lesssim E \lesssim 0.09 t$. When the corresponding density of states (DOS) is analyzed [cf. Figure 4b], one identifies two sharp peaks at $E=0$ and $E=0.09 t$ arising from the formation of strain-induced LL with $n=0,1$. From these, we extract a PMF $B_{s} \approx 65 \mathrm{~T}$ and this estimate allows us to further confirm that local maxima occurring in the conductance of Figure $4 \mathrm{a}$ correlate to higher energy LL $\left(E_{2}=0.13 t, E_{3}=0.16 t\right.$ and $\left.E_{4}=0.18 t\right)$. The dependence of the conductance on device size for the same spatial distribution of PMF can be studied without having to run a new shape optimization procedure by exploring the scaling implied by eq. (11). For example, if one scales the inner and outer boundaries as $R_{\text {in }}^{\alpha}(\theta)=\alpha R_{\text {in }}(\theta)$, $R_{\text {out }}^{\alpha}(\theta)=\alpha R_{\text {out }}(\theta)$, these relations can be used to extract the pressure needed to achieve the same PMF pattern, as well as the magnitude of the quasi-uniform field (see Fig. S5 and related discussion in the Supporting Information). The conductance of a device thus scaled with $\alpha=3\left(R_{\text {out }} \simeq 80 \mathrm{~nm}\right)$ is shown in Figure 4 for maximal strains of $\varepsilon_{\mathrm{m}}=2.48 \%$ and $\varepsilon_{\mathrm{m}}=5.16 \%$. In the two cases both the plateau and resonant peak are again present, from which we estimate $B_{s} \approx 9 \mathrm{~T}$ and $B_{s} \approx 20 \mathrm{~T}$, respectively. However, in larger devices these features are much more sharply defined and the plateau is flatter: the conductance is therefore more perfectly quan- tized at $6\left(2 e^{2} / \hbar\right)$ with growing device dimension. They also require less strain to emerge due to the fact that the inner and outer contacts become decoupled at lower PMF (because the condition that the magnetic length, $\ell_{B}=\sqrt{\hbar / e B}$, is much less than $L$ is met at smaller PMF).

\section{Perfectly conducting channels and valley filter- ing}

In order to understand the origin of this robust quantization induced by pressure over a large range of energies that are experimentally relevant, let us look first at the real-space distribution of the electronic wavefunctions. The local DOS (LDOS) calculated at the plateau midpoint $(E=0.04 t)$ and at the peak $(E=0.09 t)$ are shown in Figures $4 \mathrm{~d}$ and 4, respectively, for the device with $\alpha=1$ pressurized to $\varepsilon_{\mathrm{m}}=6.11 \%$ [cf. Figure 4 4 ]. At the peak [ $E=0.09 t$, Figure [4], the observation of a state entirely confined in the central portions of the device identifies it as clearly associated with one of the PMF-induced LLs. ${ }^{19}$ In sharp contrast, for energies in the plateau [ $E=0.04 t$, Figure 4 $]$ ] the LDOS concentrates on six narrow radial channels of the flower device. Comparing the LDOS with the PMF map shown in Figure 2(b) reveals that the wavefunction concentrates at precisely - and only - those regions where the PMF changes polarity. Consequently, the plateau in conductance at low energies is associated with current being carried by these pseudomagnetic interfacial states, similarly to the corresponding situation in nonuniform (real) magnetic fields ${ }^{30}$ where electrons propagate chirally along a boundary separating fields 
a

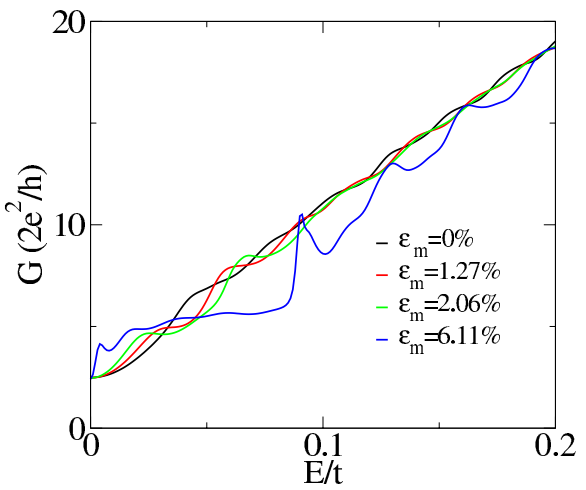

d

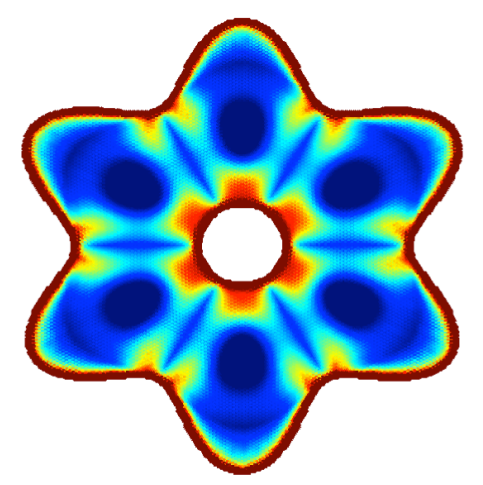

b

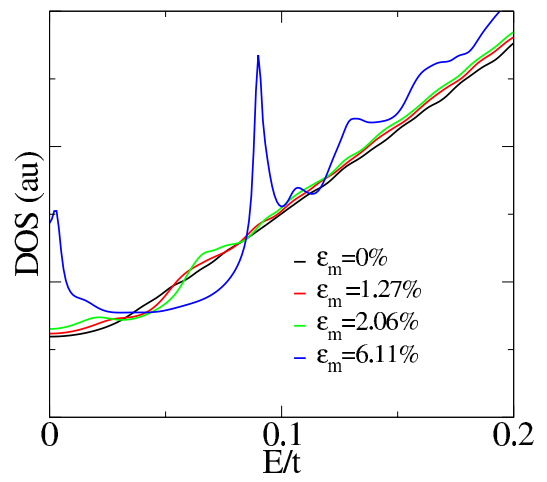

e

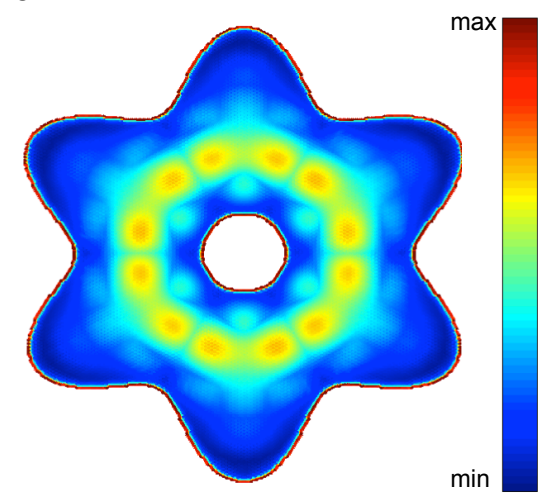

C

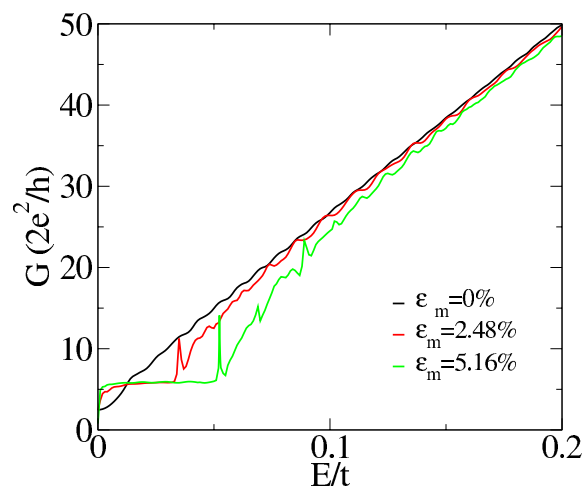

f

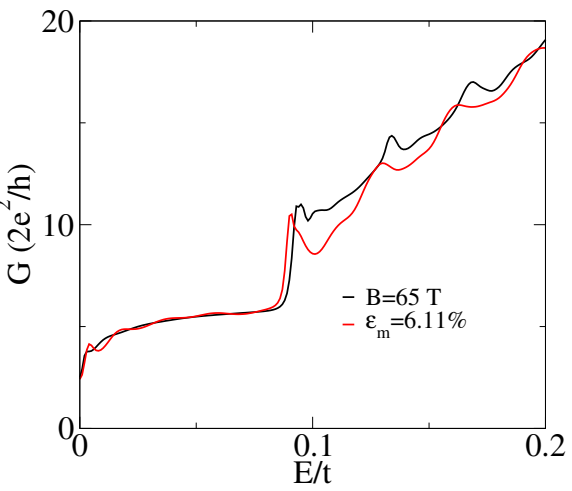

Figure 4: (color online) The top row shows plots of the conductance (a) and the DOS (b) of a device with $R_{\text {in }} \approx 4.7 \mathrm{~nm}$ and $20 \mathrm{~nm} \leq R_{\text {out }} \leq 32.2 \mathrm{~nm}(\alpha=1)$ for different values of maximal strain $\varepsilon_{\mathrm{m}}$, as well as the conductance of a similar device scaled with $\alpha=3$ for different values of maximal strain (c). The bottom row shows the LDOS at $E=0.04 t$ (d) and $E=0.09 t$ (e) of the device in (a). (f) Comparison of the conductance of the flower device used in (a) for $\varepsilon_{\mathrm{m}}=6.11 \%$ with that of an unpressurized one, where the latter includes a real magnetic field $(B=65 \mathrm{~T})$ with a simulated spatial pattern that matches the one of the PMF [see also Figure 5 a].

of opposite polarity.

A useful picture of the nature of these 1D modes stabilized at the polarity inversion interfaces is provided by the semi-classical limit of this problem where they become socalled snake states. $\frac{31}{11}$ The designation arises from the different winding sense of the electron orbits in regions where $B$ has opposite polarity, which allows them to be confined and propagate along the interface with a definite direction. This interpretation helps one to understand: (i) directionality of the states and (ii) localization in regions where $B_{s} \approx 0$ and changes sign. However, despite the usefulness of the semiclassical perspective, it is important to retain that no clear correspondence to the classical motion can be established in the case shown in Figure $4 \mathrm{~d}$ because, as we are looking at energies between the $n=0$ and $n=1 \mathrm{LL}$, the cyclotron radius is smaller than the magnetic length for the energies

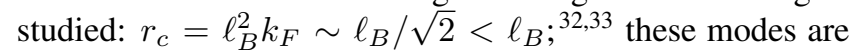
in the quantum regime.

At low-energies, transport takes place via one-dimensional perfectly conducting modes, which is entirely consistent with the 1D-like energy dependence of the DOS plotted in Figure $4 \mathrm{~b}$ for $\varepsilon_{\mathrm{m}}=6.11 \%$. Moreover, the six neighbor- ing sectors of alternating PMF polarity in our flower device are expected to beget six snake state channels, so one may deduce the existence of a plateau in the conductance of $6\left(2 e^{2} / h\right)$, which is very approximately the case seen in Figures $4 \mathrm{a}$ and $4 \mathrm{c}$. Note that, while one might think that the difference between the observed $\left[5.7\left(2 e^{2} / h\right)\right]$ and theoretical values $\left[6\left(2 e^{2} / h\right)\right]$ of the conductance plateau is due to the inhomogeneity of PMF over each sector, a direct comparison between the conductance of the pressurized device with an unpressurized flower under a real magnetic field with artificially sharp polarity changes shows no difference. More specifically, we have calculated the conductance of the same unpressurized device with an external magnetic field of constant magnitude $(B=65 \mathrm{~T})$ but alternating in sign at the six places where the PMF does so [cf. Figure 2(b) and Figure 5a] (additional details and different cases are discussed in the Supporting Information). Figure $4 \mathrm{f}$ demonstrates that the conductance of the pressurized device with $\varepsilon_{\mathrm{m}}=6.11 \%$ is essentially the same as that of the idealized unpressurized device with real magnetic field of the same magnitude. The agreement is nearly perfect in the plateau region below the first LL, further corroborating that the flower device is the 
optimal solution (to emphasize this point, in the supplementary Fig. S4(e) we show that the conductance of pressurized annular devices that retain a perfectly circular shape does not develop the quantization plateau). It also validates our earlier argument regarding the origin of the chiral 1D channels in the pressurized devices and their close analogy to the semi-classical snake states. In the end, we can trace the small deviation from the ideal $6\left(2 e^{2} / h\right)$ quantization in the pressurized devices of Figure 4 to a small coupling between the modes belonging to different radial segments: to avoid such coupling requires $R_{\text {in }} \gtrsim 2 \ell_{B}$ so that the wave functions of neighboring modes do not overlap near the inner contact, whereas this device has $R_{\text {in }} \approx 4.7 \mathrm{~nm}$ and $\ell_{B}=3.1 \mathrm{~nm}$ which falls short of it. In the supplementary Fig. S4 we show that perfect quantization is indeed achieved when $R_{\text {in }}$ and $\ell_{B}$ fulfill that criterion and that, as expected more generally, the conductance step is quantized at $G=2 n_{s} e^{2} / h$, where $n_{s}$ is the number of polarity changing interfaces in the disk.

Given this scenario, the way in which PMFs couple to the electronic motion in graphene has an interesting and useful implication. Since electrons belonging to distinct valleys feel PMFs with opposite sign, 45 they propagate in opposite directions along the polarity boundary of the field. This effect is schematically illustrated in Figures 5 a for an electron from valley $K$, and 5 p for valley $K^{\prime}$. Looking back at Figure $4 \mathrm{~d}$, each radial segment along which the LDOS is peaked supports two chiral 1D modes, each propagating in opposite radial directions, and each associated with one given valley. Suppose then that the outer contact does not cover the entire perimeter but is, instead, sectioned into six outer contacts that allow collection of current only from the vicinity of the $1 \mathrm{D}$ channels (for our geometries that would mean defining contacts in the vicinity of the indentations of the outer perimeter, as in Figures $5 \mathrm{a}$ and $5 \mathrm{~b}$ ). Under a given bias between inner and outer contacts (say, electrons flowing radially outward), the electrons arising from valley $K$ would reach three specific contacts (all equivalent, oriented $2 \pi / 3$ apart from each other), whereas those associated with valley $K^{\prime}$ would reach the other three contacts, as indicated in Figures 5 and $5 \mathrm{~b}$. In this schematic, electrons from valley $K$ are channeled by the 1D modes from the inner contact "I" to the outer contacts 1,2 and 3, while those from valley $K^{\prime}$ can only reach contacts 4,5 and 6 . A change in the bias sign, or changing from electron to hole-doped graphene with a back gate, would exchange the valleys that "reach" a given contact. In this manner, the device can spatially separate individual contributions from each valley and deliver valley polarized current to specific contacts or, alternatively, selectively filter or probe the existence of valley polarized currents.

We highlight that the observation of these effects in our flower-like geometry is not limited to the specific range of hydrostatic pressure and device dimensions used for the calculations reported in Figure 5. For example, using the scaling relations 11 , we expect that scaling up the device to $L=400 \mathrm{~nm}$ while preserving its shape and using a pressure of $\sim 5.5$ bar shall create a PMF of $\sim 0.26 \mathrm{~T}$ with the same spatial distribution. The valley filtering effect will take place as long as LLs can be formed in the center of each petal guaranteeing that transport only takes place via the $1 \mathrm{D}$ modes. A simple estimate of the electronic mobilities needed for this can be made from the criterion that the mean free path, $\ell$, be larger than the characteristic cyclotron diameter: $\ell \gtrsim 2 \ell_{B}$. In terms of the mobility defined as $\mu=\ell e / \hbar k_{F}$ and using $k_{F}=\sqrt{2} / l_{B}$ for electrons in the first LL, this becomes $\mu \gtrsim 2 \ell_{B} e / \hbar k_{F}=\sqrt{2} \ell_{B}^{2} e / \hbar \sim 5 \times 10^{4} \mathrm{~cm}^{2} / \mathrm{V} \mathrm{s}$, where we used $l_{B}=\sqrt{\hbar / e B} \approx 50 \mathrm{~nm}$; such mobilities are rather standard in graphene devices (see Fig. S6 and related discussion in the Supporting Information for additional details).

Recent research has shown the existence of snake states in strained graphene nanoribbons, and diverse valley filtering devices have been proposed by exploiting strained graphene. ${ }^{34}$ Similarly to ours, these devices require a specific geometry. However, ours does not require external electric or magnetic fields, produces valley filtered currents equally for both valleys, and whether the filtering occurs or not can be controlled by pressure or strain.

\section{Effects of disorder and high on/off ratios}

As often happens, disorder can simultaneously be detrimental or functional in solid state systems. From the first point of view, the conductance plateau associated with the snake states should be affected by disorder in real samples. As each radial channel supports one pair of opposite chirality and opposite valley quantum number, short-range scatterers will be particularly disadvantageous since they cause intervalley scattering and, because of the valley-chirality locking, that translates into backscattering and the degradation of their perfectly conducting nature. Additionally, the snake states' locations coincide with the sites of highest curvature in the pressurized flower where the probability of adsorbing alien atoms or molecules is higher. ${ }^{4041}$ In order to assess this in a specific manner, we modeled the attachment of adatoms to graphene in these regions by adding an Anderson-impurity type perturbation to the electronic Hamiltonian: each carbon can bind an adsorbate with probability $p_{a d}$; this introduces a new electronic level of energy $E_{a d}=t / 16$ that the electron can hop to from the attached carbon with a hopping amplitude $V=2 t$ that describes the degree of hybridization. ${ }^{42}$ The conductance averaged over an ensemble of 20 such systems with $p_{a d}=0.1$ for the device of Figure $4 a$ is plotted in Figure 57. As anticipated, the presence of these dopants in the regions where the snake states occur increases backscattering, thus lowering the conductance. The plateau is seen to remain, but its height depends inversely on the size of the system (not shown); in this particular case, traces of quantization are still present due to the small width of the device used ( $W_{\text {eff }} \approx 223 \AA$ ). We point out that the approximate preservation of the plateau under strong disorder (it affects $10 \%$ of the atoms) is further evidence of the 1D character and resilience of the underlying modes: there is now backscattering (the plateau has smaller $G$ ), but the onedimensionality is unaffected (a plateau largely remains).

Whereas such a system with strong short-range scattering will be less effective as a valley filter, the sensitivity of the low-energy base conductance to the radial dimension can have practical functional applications, as we now describe. 
a

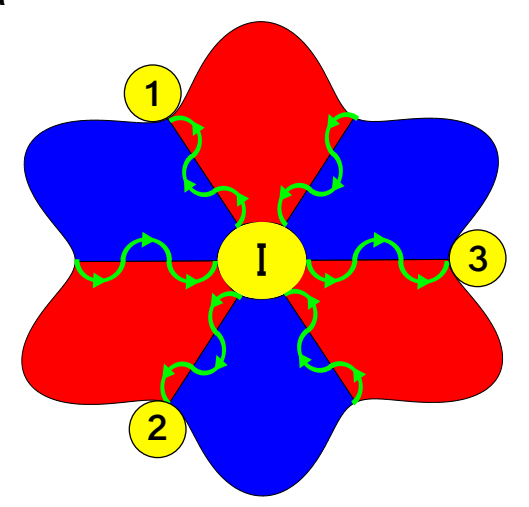

C

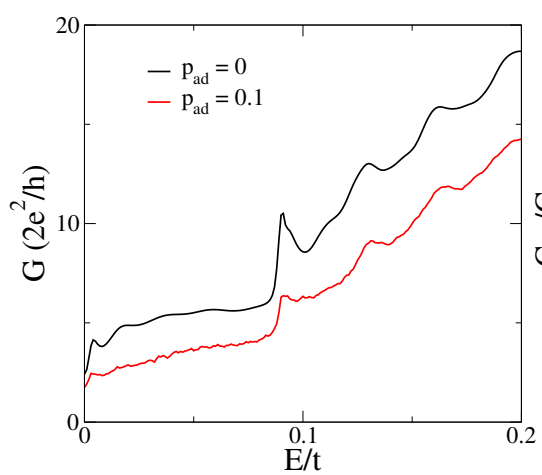

b

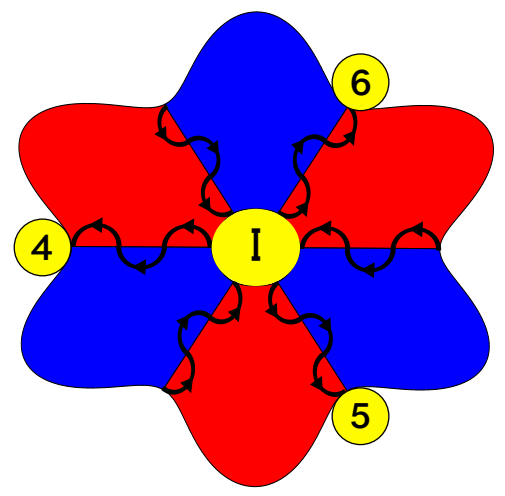

d

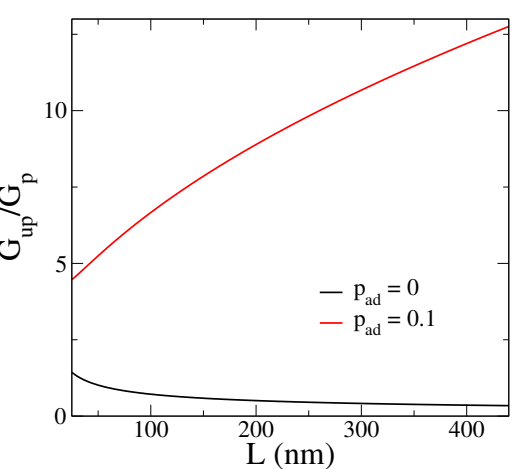

Figure 5: (color online) Schematic distribution and polarity of $B_{s}$, as well as the direction of propagation of the snake states, for electrons in valley $K$ (a) valley $K^{\prime}$ (b). In our device, transport occurs via transmission from the inner contact (I) to the six outer contacts labeled $1 \ldots 6$, where 1-3 collect electrons from valley $K$ only (panel a), and 4-6 from valley $K^{\prime}$ (panel b). (c) Conductance of the pressurized $\alpha=1$ flower device when the probability of adsorbing adatoms onto graphene is $p_{a d}=0.0$ (clean) and $p_{a d}=0.1$ (disordered). (d) The unpressurized/pressurized conductance ratio $\left(G_{\mathrm{up}} / G_{\mathrm{p}}\right)$ as a function of the device's linear dimension $(L)$ for $p_{a d}=0.0$. This ratio is computed at the midpoint between the 0th and 1st Landau level energies of the pressurized device.

Under pressure, the perfectly conducting channels limit the minimum conductance that can be achieved, irrespective of how large the magnitude of the PMF might be. From this standpoint, they are leaky electronic devices without an off state. But the residual conductance can be controlled by the type and amount of disorder, suggesting a tuneable on-off ratio. To simulate larger devices without incurring a high computational cost, and given that all of the above establishes the one-dimensional character of transport at low energies, we resort to a simple one-dimensional model ${ }^{[43}$ to scale up the conductance for larger pressurized disordered devices:

$$
G_{\mathrm{p}}\left(W_{\text {eff }}\right)=6 \mathcal{T}\left(\frac{2 e^{2}}{h}\right), \quad \mathcal{T}=\frac{T}{T+(1-T) N} .
$$

This assumes that in the snake-type 1D modes, electrons transmit through one adatom with probability $T$ and $N=$ $\left(W_{\text {eff }} / a\right) p_{a d}$ is the number of scatterers in the channel of width $W_{\text {eff. }}$ It follows that wide devices should have a notorious reduction of the conductance, entailing high ratios of unpressurized/pressurized conductance $\left(G_{\mathrm{up}} / G_{\mathrm{p}}\right)$ in the energy region of the snake states. This ratio can be used as a figure of merit directly related to the on-off ratio of the device. To quantify $G_{\text {up }} / G_{\mathrm{p}}$, we employed a number of modeling assumptions. Firstly, the Fermi energy was fixed at $E_{F}=E_{1} / 2$, corresponding to one half of the energy of the $n=1 \mathrm{LL}$, the midpoint of the conductance plateau. Secondly, a perfectly round inner contact and ballistic transmission were considered in order to estimate $G_{\text {up }}=\left(4 e^{2} / h\right) 2 j_{m}$, where $j_{m} \sim k_{F} R_{\text {in }}$ is the maximum total angular momentum. ${ }^{[25]}$ Third, geometrical factors such as $R_{\text {in }}$ and $W_{\text {eff }}$ were scaled from our original device $(\alpha=1)$. Finally, the value $T \approx 0.9$ was extracted from the disordered conductance of Figure 5, from where we obtain $\mathcal{T} \approx 0.6$. Figure $5 \mathrm{~d}$ shows that, in pristine devices, $G_{\text {up }} / G_{\mathrm{p}}$ decreases as $\propto L^{-3 / 2}$. This decrease is due solely to $G_{\text {up }}$ because, in a clean device, $G_{\mathrm{p}}$ remains at the value $\approx 6\left(2 e^{2} / h\right)$ for any $L$ as long as $E_{F}=E_{1} / 2$. But, since the PMF scales inversely with $L$ for a fixed device shape, to keep this choice of $E_{F}$ requires its value to vary for different $L$. Hence, each $L$ in the figure corresponds to a different $k_{F}$, and these parameters vary inversely to each other; this causes the overall scaling shown by the black line in the figure. However, in the presence of the adsorbates, the 1D transport regime that occurs 
under pressure is expected to be much more sensitive to the disorder. The rapid decrease of the transmission probability $\mathcal{T}$ with $L$ in this case dominates over the variation of $G_{\text {up }}$. As a result, the figure of merit, which is governed by the overall transmission probability through $G_{\text {up }} / G_{\mathrm{p}} \propto j_{m} / \mathcal{T}$, increases with the channel length reaching values near 12 for a device with $L=400 \mathrm{~nm}\left(R_{\text {in }}=70 \mathrm{~nm}, \alpha=15\right)$, a high "on/off" value considering the absence of a band gap in graphene.

\section{Conclusions}

In this work we set out to determine the optimal geometry of a graphene Corbino device that guarantees a PMF of nearly constant magnitude throughout most of the system when externally pressurized. Since the sign of the PMF has to inevitably alternate six times along any closed path containing the inner contact, the field cannot be strictly constant in the whole ring. ${ }^{8}$ Yet, both its magnitude and absolute value can be made satisfactorily uniform. The first case is discussed in the Supporting Information and we see that it is possible to require a specific sign and magnitude in most of the system, and the effect of the optimization process is to generate a geometry where the regions with the opposite PMF are much reduced (a more extreme spatial reduction is possible under higher pressures and higher target PMFs). If, on the other hand, the goal is only the PMF magnitude, but not its sign, the optimal geometries are the sixfold flower shapes such as in Figure 2 that we have analyzed in detail. The spatial boundaries where the PMF changes sign for the latter are sharper and better defined, which leads to robust snake states and strictly one-dimensional transport over a range of pressures. This radially one-dimensional regime is signaled by the strict quantization of the conductance at $2 e^{2} / h$ per channel that is seen to survive up to high values of $E_{F}$ (e.g. 50-100 meV, cf Figure 4), the characteristically 1D behavior of the DOS as a function of energy there, and corroborated by the real space profile of the LDOS.

The strain-induced perfectly conducting channels can be exploited in two different directions for electronic applications. On the one hand, they limit from below the current pinch-off effect and make these devices leaky because of their chiral nature. Our studies of the effect of disorder show that short-range defects can activate inter-valley scattering and reduce the residual conductance. Since their spatial location is set by the geometry, we can envisage this being done in a deliberate way through adsorbates, for example, so that only the regions where snake states develop under pressure become disordered. In this way, the conductance in the unpressurized state would remain unaffected, but the perfectly conducting channels would no longer exist under pressure which would significantly boost the on/off ratio. The pressure sensitivity and its direct translation into current modulations, suggests its possible application in electromechanical sensing or transducers. ${ }^{44}$ On the other hand, we have also seen an equally interesting perspective where snake-type states and their leaky residual conductance are not detrimental but, instead, functional: clean de- vices can be employed as sources of valley-polarized currents in graphene. Note that the Corbino ring (the device) is defined only by the indentation of the substrate, not by an actual patterning of graphene, and the inner and outer contacts at $R_{\text {in }}$ and $R_{\text {out }}$ are still the same sheet of graphene. Hence, the sheet can extend over large distances beyond the outer radius and these structures can act as local sources of valley-polarized currents for injection into the two dimensional graphene plane beyond $R_{\text {out }}$.

Acknowledgement DAB acknowledges the support from Mackpesquisa and FAPESP under grant 2012/50259-8, VMP that of the Singapore Ministry of Education Academic Research Fund Tier 2 under grant number MOE2015-T2-2059, and AHCN the support of the National Research Foundation of Singapore under the Mid Size Centre Grant. Numerical computations were carried out at the HPC facilities of the NUS Centre for Advanced 2D Materials.

\section{References}

(1) Booth, T. J.; Blake, P.; Nair, R. R.; Jiang, D.; Hill, E. W.; Bangert, U.; Bleloch, A.; Gass, M.; Novoselov, K. S.; Katsnelson, M. I.; Geim, A. K. Nano Lett. 2008, 8, 2442.

(2) Lee, C.; Wei, X.; Kysar, J. W.; Hone, J. Science 2008, 321,385 .

(3) Kane, C. L.; Mele, E. J. Phys. Rev. Lett. 1997, 78, 1932.

(4) Suzuura, H.; Ando, T. Phys. Rev. B 2002, 65, 235412.

(5) Vozmediano, M. A. H.; Katsnelson, M. I.; Guinea, F. Phys. Rep. 2010, 496, 109.

(6) Pereira, V. M.; Castro Neto, A. H. Phys. Rev. Lett. 2009, 103, 046801.

(7) Tomori, H.; Kanda, A.; Goto, H.; Ootuka, Y.; Tsukagoshi, K.; Moriyama, S.; Watanabe, E.; Tsuya, D. Appl. Phys. Express 2011, 4, 075102.

(8) Guinea, F.; Katsnelson, M. I.; Geim, A. K. Nat. Phys. 2009, 6, 30-33.

(9) San-Jose, P.; González, J.; Guinea, F. Phys. Rev. Lett. 2011, 106, 045502 .

(10) Low, T.; Guinea, F.; Katsnelson, M. I. Phys. Rev. B 2011, 83, 195436.

(11) Wu, Z.; Zhai, F.; Peeters, F. M.; Xu, H. Q.; Chang, K. Phys. Rev. Lett. 2011, 106, 176802.

(12) Klimov, N. N.; Jung, S.; Zhu, S.; Li, T.; Wright, C. A.; Solares, S. D.; Newell, D. B.; Zhitenev, N. B.; Stroscio, J. A. Science 2012, 336, 1557.

(13) Lu, J.; Castro Neto, A. H.; Loh, K. P. Nat. Commun. 2012, 3, 823 . 
(14) de Juan, F.; Sturla, M.; Vozmediano, M. A. H. Phys. Rev. Lett. 2012, 108, 227205.

(15) Abanin, D. A.; Pesin, D. A. Phys. Rev. Lett. 2012, 109, 066802.

(16) Pellegrino, F. M. D.; Angilella, G. G. N.; Pucci, R. Phys. Rev. B 2011, 84, 195404.

(17) Bahamon, D. A.; Pereira, V. M. Phys. Rev. B 2013, 88, 195416.

(18) Yesilyurt, C.; Tan, S. G.; Liang, G.; Jalil, M. B. A. AIP Adv. 2016, 6, 056303.

(19) Qi, Z.; Bahamon, D. A.; Pereira, V. M.; Park, H. S.; Campbell, D. K.; Castro Neto, A. H. Nano Lett. 2013, 13, 2692-2697.

(20) Katsnelson, M. I.; Novoselov, K. S.; Geim, A. K. Nat. Phys. 2006, 2, 620.

(21) Levy, N.; Burke, S. A.; Meaker, K. L.; Panlasigui, M.; Zettl, A.; Guinea, F.; Castro Neto, A. H.; Crommie, M. F. Science 2010, 329, 544-547.

(22) Jones, G. W.; Pereira, V. M. New J. Phys. 2014, 16, 093044.

(23) Persson, P.-O.; Strang, G. SIAM Rev. 2004, 46, 329.

(24) Meir, Y.; Wingreen, N. S. Phys. Rev. Lett. 1992, 68, 2512-2515.

(25) Bahamon, D. A.; Castro Neto, A. H.; Pereira, V. M. Phys. Rev. B 2013, 88, 235433.

(26) Bahamon, D. A.; Qi, Z.; Park, H. S.; Pereira, V. M.; Campbell, D. K. Phys. Rev. B 2016, 93, 235408.

(27) Tománek, D.; Louie, S. G. Phys. Rev. B 1988, 37, 8327-8336.

(28) Pereira, V. M.; Castro Neto, A. H.; Peres, N. M. R. Phys. Rev. B 2009, 80, 045401.

(29) Yan, W.; He, W.-Y.; Chu, Z.-D.; Liu, M.; Meng, L.; Dou, R.-F.; Zhang, Y.; Liu, Z.; Nie, J.-C.; He, L. Nat. Commun. 2013, 4, 2159.

(30) Park, S.; Sim, H.-S. Phys. Rev. B 2008, 77, 075433.

(31) Müller, J. E. Phys. Rev. Lett. 1992, 68, 385-388.

(32) Rickhaus, P.; Makk, P.; Liu, M.-H.; Tóvári, E.; Weiss, M.; Maurand, R.; Richter, K.; Schönenberger, C. Nat. Commun. 2015, 6, 6470.

(33) Taychatanapat, T.; Tan, J. Y.; Yeo, Y.; Watanabe, K.; Taniguchi, T.; Özyilmaz, B. Nat. Commun. 2015, 6, 6093.
(34) Carrillo-Bastos, R.; León, C.; Faria, D.; Latgé, A.; Andrei, E. Y.; Sandler, N. Phys. Rev. B 2016, 94, 125422.

(35) Low, T.; Guinea, F. Nano Lett. 2010, 10, 3551-3554.

(36) Gunlycke, D.; White, C. T. Phys. Rev. Lett. 2011, 106, 136806.

(37) Fujita, T.; Jalil, M. B. A.; Tan, S. G. Appl. Phys. Lett. 2010, 97, 043508.

(38) Settnes, M.; Power, S. R.; Brandbyge, M.; Jauho, A.-P. Phys. Rev. Lett. 2016, 117, 276801.

(39) Milovanović, S. P.; Peeters, F. M. Appl. Phys. Lett. 2016, 109, 203108.

(40) Geim, A. K. Science 2009, 324, 1530-1534.

(41) Cretu, O.; Krasheninnikov, A. V.; RodríguezManzo, J. A.; Sun, L.; Nieminen, R. M.; Banhart, F. Phys. Rev. Lett. 2010, 105, 196102.

(42) Peres, N. M. R. Rev. Mod. Phys. 2010, 82, 2673-2700.

(43) Datta, S. Electronic Transport in Mesoscopic Systems; Cambridge University Press, 1995.

(44) Zhou, Q.; Zettl, A. Appl. Phys. Lett. 2013, 102, 223109. 


\section{Supporting Information}

\section{Quantized transport, strain-induced perfectly conducting modes and valley filtering on shape-optimized graphene Corbino devices}

Gareth W. Jones, ${ }^{\dagger}$ Dario Andres Bahamon,${ }^{\ddagger}$ Antonio H. Castro Neto,,$\S \S$ and Vitor M. Pereira*,$\$$

$\dagger$ School of Mathematics, The University of Manchester, Manchester, M13 9PL, England $\ddagger$ MackGraphe-Graphene and Nano-Materials Research Center, Mackenzie Presbyterian University, Rua da Consolação 896, 01302-907, São Paulo, SP, Brazil

\Department of Physics, National University of Singapore, 2 Science Drive 3, Singapore 117542

$\S$ Centre for Advanced 2D Materials, National University of Singapore, 6 Science Drive 2, Singapore 117546

E-mail: vpereira@nus.edu.sg

Phone: +65 66013642 


\section{Details of the optimization procedure and equations}

In dimensionless terms (signified by an overbar, ${ }^{-}$), the full optimization problem to be solved is as follows:

$$
\text { Minimize } \quad \overline{\mathcal{I}}=\frac{1}{\operatorname{area} \bar{\Omega}} \iint_{\bar{\Omega}\left[c_{1}, \ldots, c_{n}\right]}\left(\bar{B}^{2}-1\right)^{2} \mathrm{~d}^{2} \overline{\boldsymbol{X}}+\eta \mathcal{I}^{\mathrm{reg}}\left[c_{1}, \ldots, c_{n}\right]
$$

subject to the six equations (valid for all admissable variations $\tilde{*}$ )

$$
\begin{gathered}
\frac{1}{\operatorname{area} \bar{\Omega}} \iint_{\bar{\Omega}\left[c_{1}, \ldots, c_{n}\right]}\left[\frac{\partial \tilde{v}_{1}}{\partial \bar{X}} \bar{N}_{11}+\frac{\partial \tilde{v}_{1}}{\partial \bar{Y}} \bar{N}_{12}\right] \mathrm{d}^{2} \overline{\boldsymbol{X}}=0 \\
\frac{1}{\operatorname{area} \bar{\Omega}} \iint_{\bar{\Omega}\left[c_{1}, \ldots, c_{n}\right]}\left[\frac{\partial \tilde{v}_{2}}{\partial \bar{X}} \bar{N}_{12}+\frac{\partial \tilde{v}_{2}}{\partial \bar{Y}} \bar{N}_{22}\right] \mathrm{d}^{2} \overline{\boldsymbol{X}}=0 \\
\frac{1}{\operatorname{area} \bar{\Omega}} \iint_{\bar{\Omega}\left[c_{1}, \ldots, c_{n}\right]}\left\{\frac{\partial \tilde{w}}{\partial \bar{X}}\left(-\kappa\left(\frac{\partial \bar{M}_{11}}{\partial \bar{X}}+\frac{\partial \bar{M}_{12}}{\partial \bar{Y}}\right)+\bar{N}_{11} \frac{\partial \bar{w}}{\partial \bar{X}}+\bar{N}_{12} \frac{\partial \bar{w}}{\partial \bar{Y}}\right)\right. \\
\left.+\frac{\partial \tilde{w}}{\partial \bar{Y}}\left(-\kappa\left(\frac{\partial \bar{M}_{12}}{\partial \bar{X}}+\frac{\partial \bar{M}_{22}}{\partial \bar{Y}}\right)+\bar{N}_{12} \frac{\partial \bar{w}}{\partial \bar{X}}+\bar{N}_{22} \frac{\partial \bar{w}}{\partial \bar{Y}}\right)+\tilde{w} \bar{p}\right\} \mathrm{d}^{2} \overline{\boldsymbol{X}}=0 \\
\frac{1}{\operatorname{area} \bar{\Omega}} \iint_{\bar{\Omega}\left[c_{1}, \ldots, c_{n}\right]}\left[\frac{1}{\left(1-\nu^{2}\right)}\left(\bar{M}_{11}-\nu \bar{M}_{22}\right) \tilde{M}_{11}+\frac{\partial \bar{w}}{\partial \bar{X}} \frac{\partial \tilde{M}_{11}}{\partial \bar{X}}\right] \mathrm{d}^{2} \overline{\boldsymbol{X}}=0 \\
\frac{1}{\operatorname{area} \bar{\Omega}} \iint_{\bar{\Omega}\left[c_{1}, \ldots, c_{n}\right]}\left[\frac{1}{(1-\nu)} \bar{M}_{12} \tilde{M}_{12}+\frac{1}{2} \frac{\partial \bar{w}}{\partial \bar{X}} \frac{\partial \tilde{M}_{12}}{\partial \bar{Y}^{2}}+\frac{1}{2} \frac{\partial \bar{w}}{\partial \bar{Y}} \frac{\partial \tilde{M}_{12}}{\partial \bar{X}}\right] \mathrm{d}{ }^{2} \overline{\boldsymbol{X}}=0 \\
\frac{1}{\operatorname{area} \bar{\Omega}} \iint_{\bar{\Omega}\left[c_{1}, \ldots, c_{n}\right]}\left[\frac{1}{\left(1-\nu^{2}\right)}\left(\bar{M}_{22}-\nu \bar{M}_{11}\right) \tilde{M}_{22}+\frac{\partial \bar{w}}{\partial \bar{Y}} \frac{\partial \tilde{M}_{22}}{\partial \bar{Y}}\right] \mathrm{d}^{2} \overline{\boldsymbol{X}}=0
\end{gathered}
$$


together with the additional definitions

$$
\begin{gathered}
\bar{N}_{11}=\bar{\varepsilon}_{11}+\nu \bar{\varepsilon}_{22}, \quad \bar{N}_{12}=(1-\nu) \bar{\varepsilon}_{12}, \quad \bar{N}_{22}=\nu \bar{\varepsilon}_{11}+\bar{\varepsilon}_{22}, \\
\bar{\varepsilon}_{11}=\frac{\partial \bar{v}_{1}}{\partial \bar{X}}+\frac{1}{2}\left(\frac{\partial \bar{w}}{\partial \bar{X}}\right)^{2}, \\
\bar{\varepsilon}_{12}=\frac{1}{2}\left(\frac{\partial \bar{v}_{1}}{\partial \bar{Y}}+\frac{\partial \bar{v}_{2}}{\partial \bar{X}}+\frac{\partial \bar{w}}{\partial \bar{X}} \frac{\partial \bar{w}}{\partial \bar{Y}}\right), \\
\bar{\varepsilon}_{22}=\frac{\partial \bar{v}_{2}}{\partial \bar{Y}}+\frac{1}{2}\left(\frac{\partial \bar{w}}{\partial \bar{Y}}\right)^{2}, \\
\bar{B}\left[\bar{\varepsilon}^{\mathrm{rec}}\right]=\frac{\partial}{\partial \bar{Y}}\left(\frac{\bar{\varepsilon}_{11}^{\mathrm{rec}}-\bar{\varepsilon}_{22}^{\mathrm{rec}}}{2}\right)+\frac{\partial \bar{\varepsilon}_{12}^{\mathrm{rec}}}{\partial \bar{X}} .
\end{gathered}
$$

For a full derivation of this system in general, we refer the reader to reference 1 , but we will give a brief overview here for convenience. $\boldsymbol{X}=(X, Y)$ are the Cartesian coordinates of the undeformed graphene flake. The two corresponding in-plane displacements are $v_{1}$ and $v_{2}$, and $w$ is the out-of-plane deflection. These, in turn, lead to the strain components $\varepsilon_{\alpha \beta}$ through (9)-(11), and in turn to the stress resultants $N_{\alpha \beta}$ using (8). This linear stress-strain relationship only holds for small values of strain, namely up to around 5-6\%. The equations (2)-(7) are weak form representations of the standard Föppl-von Kármán relations for the deformation of an elastic plate. Though the typical statement of this law is in terms of two coupled fourth-order PDEs written in terms of a stress function, we do not use this representation here since the boundary conditions are displacement-based rather than forcebased. Furthermore, the advantage of a weak-form formulation is that it is straightforward to discretize using finite element methods. Throughout this investigation we used linear finite elements on a triangular discretization of the material domain $\bar{\Omega}$; this means that the variables in question are parametrized by their values at the nodal points of the mesh, and their values in the interiors of the triangular elements are suitable linear interpolations of their values at the element nodes. ${ }^{2}$

In a standard bending plate theory, the bending moments of the plate at any point are assumed to be proportional to the plate curvature, which is in turn assumed to be the 
second gradient of the out-of-plane displacement $w$. This is not achievable using linear finite elements, but we can keep the simplicity of this framework by rewriting the plate equations as a mixed variational principle, whereby the moment tensor components $M_{11}, M_{12}, M_{22}$ are assumed to be independent state variables in addition to the usual displacement variables (for full details, please see ${ }^{1}$ ). The equations are formulated to represent clamped boundary conditions, assuming that all three displacement components are zero on the boundary of $\bar{\Omega}$.

The leading-order term for the pseudomagnetic field $\bar{B}$ is given by a gradient of the strain tensor. However, the strain tensor defined by (9)-(11) is discontinuous if linear finite elements are used, and so its gradient would be undefined. Thus we must reconstruct a continuous strain field $\varepsilon_{\alpha \beta}^{\text {rec }}$ from this discontinuous data, a process known as strain recovery $\left(\right.$ see $\left.^{1,3}\right)$, and it is this which is differentiated to provide the PMF in (12).

The main goal of the method is to minimize the quantity $\overline{\mathcal{I}}$ in (1), which balances two effects: firstly we wish the square of the scaled PMF to be as close to unity as possible, which has the effect of penalizing shapes which produce large areas of near-zero PMF. The second term is a regularization term which penalizes intricate high-resolution oscillations in the outline shape (to which optimization techniques have a tendency to naturally approach), and produces smoother outlines. The regularization parameter $\eta$ tells us how strongly to weight the smoothness criterion. In our calculations we fixed this numerical constant to $10^{-5}$.

In short, the method demands that we vary the control variables $c_{1}, \ldots, c_{n}$ that define the shape, together with the state variables $\bar{v}_{1}, \bar{v}_{2}, \bar{w}, \bar{M}_{11}, \bar{M}_{12}, \bar{M}_{22}$ in such a way that the objective function $\overline{\mathcal{I}}$ is minimized subject to the elasticity constraints $(2)-(7)$. The boundary conditions applied to the system are that the displacements are set to zero on nodes comprising the inner and outer boundaries. At the boundaries $\theta= \pm \pi / 3$, we impose rotated periodicity conditions on these quantities (expressed in polar coordinates $R, \theta$ ), i.e. 
$\left.\bar{w}\right|_{\theta=\pi / 3}=\left.\bar{w}\right|_{\theta=-\pi / 3}$, and similarly for $\bar{v}_{R}, \bar{v}_{\theta}, \bar{M}_{R R}, \bar{M}_{R \theta}, \bar{M}_{\theta \theta}$. This corresponds to

$$
\begin{gathered}
\left.\bar{w}\right|_{\theta=\pi / 3}=\left.\bar{w}\right|_{\theta=-\pi / 3} \\
\left.\left(\begin{array}{cc}
1 & \sqrt{3} \\
-\sqrt{3} & 1
\end{array}\right)\left(\begin{array}{c}
\bar{v}_{1} \\
\bar{v}_{2}
\end{array}\right)\right|_{\theta=\pi / 3}=\left.\left(\begin{array}{cc}
1 & -\sqrt{3} \\
\sqrt{3} & 1
\end{array}\right)\left(\begin{array}{c}
\bar{v}_{1} \\
\bar{v}_{2}
\end{array}\right)\right|_{\theta=-\pi / 3} \\
\left.\left(\begin{array}{ccc}
1 & 2 \sqrt{3} & 3 \\
-\sqrt{3} & -2 & \sqrt{3} \\
3 & -2 \sqrt{3} & 1
\end{array}\right)\left(\begin{array}{c}
\bar{M}_{11} \\
\bar{M}_{12} \\
\bar{M}_{22}
\end{array}\right)\right|_{\theta=\pi / 3}=\left.\left(\begin{array}{ccc}
1 & -2 \sqrt{3} & 3 \\
\sqrt{3} & -2 & -\sqrt{3} \\
3 & 2 \sqrt{3} & 1
\end{array}\right)\left(\begin{array}{c}
\bar{M}_{11} \\
\bar{M}_{12} \\
\bar{M}_{22}
\end{array}\right)\right|_{\theta=-\pi / 3}
\end{gathered}
$$

in terms of Cartesian components. The same conditions hold for the variations $\tilde{v}_{1}, \ldots, \tilde{M}_{22}$, so that (for example) the equations for the moment tensor at $\theta=\pi / 3$ include relevant contributions from the boundary at $\theta=-\pi / 3$.

To recover the physical values of the variables from their equivalent dimensionless quantities $^{-}$, let $L$ be a typical length scale of the domain $\Omega, B_{0}$ to be the target PMF value, and $C$ and $D$ to be the stretching and bending moduli, respectively. We will invariably set $L$ to be the midpoint of the limits for the outer radius of the device, i.e. $L_{\text {out }}$ from equation (5) of the main text. Set

$$
\varepsilon=\frac{a e B_{0} L}{\hbar c}
$$

to be the typical scaling of the strain field, where $a=1.42 \AA$ is the interatomic spacing of the graphene lattice, $e=1.60 \times 10^{-19} \mathrm{C}$ is the elementary charge, and $c \approx 3.37$ is a dimensionless parameter related to the rate of change in the electronic hopping in graphene. ${ }^{1}$ The physical 
quantities are then written in terms of their dimensionless values through

$$
\begin{gathered}
(X, Y)=L(\bar{X}, \bar{Y}), \quad v_{\alpha}=L \varepsilon \bar{v}_{\alpha}, \quad w=L \sqrt{\varepsilon} \bar{w} \\
\varepsilon_{\alpha \beta}=\varepsilon \bar{\varepsilon}_{\alpha \beta}, \quad \varepsilon_{\alpha \beta}^{\mathrm{rec}}=\varepsilon \varepsilon_{\alpha \beta}^{\mathrm{rec}}, \quad N_{\alpha \beta}=C \varepsilon \bar{N}_{\alpha \beta}, \quad M_{\alpha \beta}=\frac{D \sqrt{\varepsilon}}{L} \bar{M}_{\alpha \beta}, \\
p=\frac{C \varepsilon^{3 / 2}}{L} \bar{p}, \quad B=B_{0} \bar{B} .
\end{gathered}
$$

Note that, in dimensional terms, the objective function (1) becomes

$$
\mathcal{I}=\frac{1}{\operatorname{area} \Omega} \iint_{\Omega\left[c_{1}, \ldots, c_{n}\right]}\left(B\left[\varepsilon^{\mathrm{rec}}\right]^{2}-B_{0}^{2}\right)^{2} \mathrm{~d}^{2} \boldsymbol{X}+\eta \mathcal{I}^{\mathrm{reg}}\left[c_{1}, \ldots, c_{n}\right]
$$

confirming that $B_{0}$ does indeed correspond to the target PMF. Also, we note that we could, in principle, replace the PMF term in the objective function by a higher power such as $\left(B^{2}-B_{0}^{2}\right)^{4}$, but this leads to a loss of sensitivity in the optimization procedure for values of $B$ near the target value $B_{0}$.

Other than Poisson's ratio $\nu$ and the dimensionless pressure $\bar{p}$, the only remaining physical parameter in the system is the dimensionless bending stiffness $\kappa$ :

$$
\kappa=\frac{D}{C L^{2} \varepsilon}=\frac{D \hbar c}{C L^{3} a e B_{0}}
$$

The bending modulus $D$ of a hexagonal carbon lattice was calculated ab initio by Kudin et al., ${ }^{4}$ who found a value of $D=1.46 \mathrm{eV}=2.34 \times 10^{-19} \mathrm{Nm}$ which will be used here. To calculate the stretching modulus $C=E h /\left(1-\nu^{2}\right)$, we use the results of Wei et al., ${ }^{5}$ who fitted a polynomial stress-strain relation to ab initio calculations up to strains of 50\%. Their linear terms are, in our notation,

$$
C=358.1 \mathrm{Nm}^{-1}, \quad C \nu=60.4 \mathrm{~N} \mathrm{~m}^{-1} \quad \Rightarrow \quad \nu=0.169
$$

Finally, note that though the method only provides the values of the graphene sheet's 
displacement at the nodal values, it is trivial to calculate the piecewise linear interpolation of these nodal values to determine the displacement of an arbitrarily-positioned atom in the lattice. We work within the Cauchy-Born framework, where macroscopic displacements are directly mapped to all the atoms in the lattice (see, e.g., references 6,7 for a discussion of how to integrate corrections arising from non-zero displacements within the crystal unit cell).

\section{Optimized shapes for different values of dimensionless bending stiffness}

As noted in the main text, and from eqs. (16), (19) 1 and (21), the optimized shapes depend only on the dimensionless quantities

$$
\bar{p}=\frac{1}{C}\left(\frac{\hbar c}{a e}\right)^{3 / 2} \frac{p}{B_{0}^{3 / 2} L^{1 / 2}}, \quad \kappa=\left(\frac{D}{C} \frac{\hbar c}{a e}\right) \frac{1}{L^{3} B_{0}} .
$$

In fact, if the bending stiffness $\kappa$ is varied while keeping $\bar{p}$ constant, there is very little variation in the shape. This is shown explicitly in Figure S1 that compares the optimal shapes obtained for different stiffness values spanning two orders of magnitude while keeping the other parameters as in the cases discussed in main text: $\bar{p}=0.337, R_{\text {out }}$ allowed to vary in the range $261 \AA \pm 60 \AA$, and $R_{\text {in }}$ in the range $61 \AA \pm 15 \AA$.

\section{Penalizing negative PMF}

It is interesting to note what happens if, instead of minimizing the integral of $\left(B^{2}-B_{0}^{2}\right)^{2}$ over the domain $\Omega$, we choose an integrand $\left(B-B_{0}\right)^{2}$. With this expression, the solution must not only have the correct magnitude of PMF, but it must also penalize negative PMF values. Employing the same initial parameters used for the flower solution $\left(B_{0}=10 \mathrm{~T}, p=10^{7} \mathrm{~Pa}\right.$, $L=261 \AA$ ), we find that the solution is a triangular-shaped flake with an inverted interior 
(a)

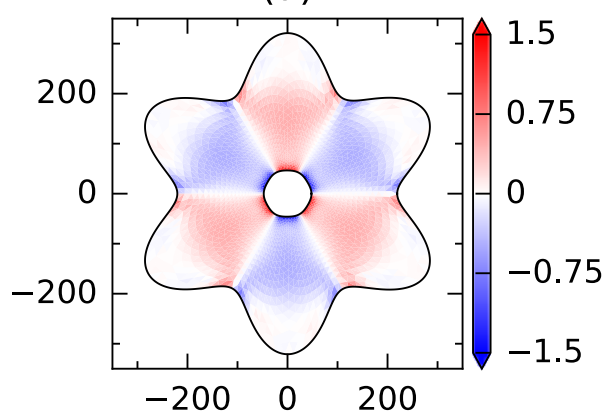

(b)

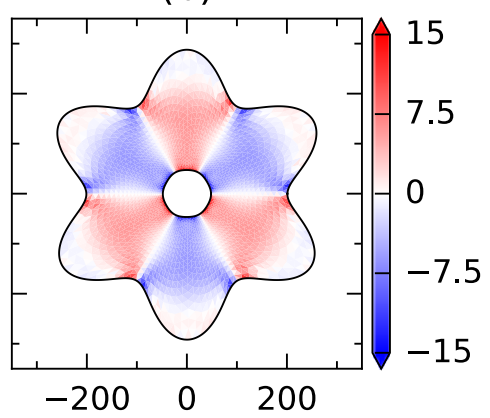

(c)

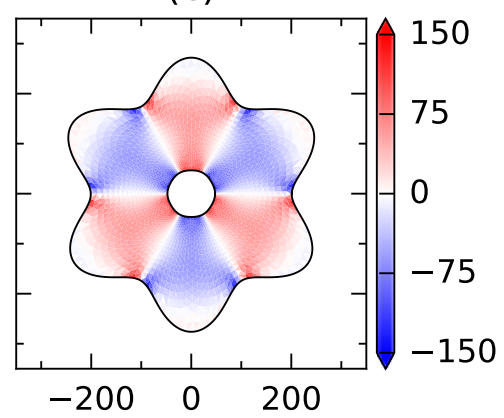

Figure S1: (color online) Output shapes at constant $\bar{p}=0.337$, while $\kappa$ takes one of the three values $\kappa \in\left\{5.76 \times 10^{-4}, 5.76 \times 10^{-5}, 5.76 \times 10^{-6}\right\}$ in plots (a), (b), (c) respectively. Physical parameters used were, respectively, $p \in\{\sqrt{10}$ bar, $100 \mathrm{bar}, \sqrt{10} \mathrm{kbar}\}$ and $B_{0} \in$ $\{1 \mathrm{~T}, 10 \mathrm{~T}, 100 \mathrm{~T}\}$. Lengths are measured in $\AA$ and PMFs in $\mathrm{T}$.

triangle, as displayed in Figure S2. It is clear that the optimization scheme has maximized the areas near $\theta \in\{\pi / 2,-\pi / 6,-5 \pi / 6\}$ where the PMFs are positive, and minimized the corresponding negative PMF regions around $\theta \in\{5 \pi / 6, \pi / 6,-\pi / 2\}$.

\section{Annular Corbino geometries}

For comparison, we calculated the PMF associated with a circular annulus, i.e. $R_{\text {out }}(\theta) \equiv$

$R_{\text {out }}=1$ and $R_{\text {in }}(\theta) \equiv R_{\text {in }}$. In this case, the system of PDEs reduces to two ODEs by symmetry:

$$
\begin{gathered}
\kappa\left[\bar{w}^{\prime \prime \prime \prime}(\bar{R})+\frac{2 \bar{w}^{\prime \prime \prime}(\bar{R})}{\bar{R}}-\frac{\bar{w}^{\prime \prime}(\bar{R})}{\bar{R}^{2}}+\frac{\bar{w}^{\prime}(\bar{R})}{\bar{R}^{3}}\right]-\bar{w}^{\prime \prime}(\bar{R})\left[\bar{v}^{\prime}(\bar{R})+\frac{\left(\bar{w}^{\prime}(\bar{R})\right)^{2}}{2}+\frac{\nu \bar{v}(\bar{R})}{\bar{R}}\right] \\
-\frac{\bar{w}^{\prime}(\bar{R})}{\bar{R}}\left[\nu \bar{v}^{\prime}(\bar{R})+\frac{\nu\left(\bar{w}^{\prime}(\bar{R})\right)^{2}}{2}+\frac{\bar{v}(\bar{R})}{\bar{R}}\right]+\bar{p}=0, \\
\bar{v}^{\prime \prime}(\bar{R})+\frac{\bar{v}^{\prime}(\bar{R})}{\bar{R}}-\frac{\bar{v}(\bar{R})}{\bar{R}^{2}}+\bar{w}^{\prime}(\bar{R}) \bar{w}^{\prime \prime}(\bar{R})+\frac{(1-\nu)\left(\bar{w}^{\prime}(\bar{R})\right)^{2}}{2 \bar{R}}=0
\end{gathered}
$$

with $\bar{v}=\bar{w}=\bar{w}^{\prime}=0$ on $\bar{R}=R_{\text {in }}$ and $\bar{R}=1$. Then the dimensionless PMF is given by

$$
\bar{B}=\frac{\sin 3 \theta}{2}\left[-\frac{(3-\nu)\left(\bar{w}^{\prime}(\bar{R})\right)^{2}}{2 \bar{R}}-\frac{4 \bar{v}^{\prime}(\bar{R})}{\bar{R}}+\frac{4 \bar{v}(\bar{R})}{\bar{R}^{2}}\right]
$$




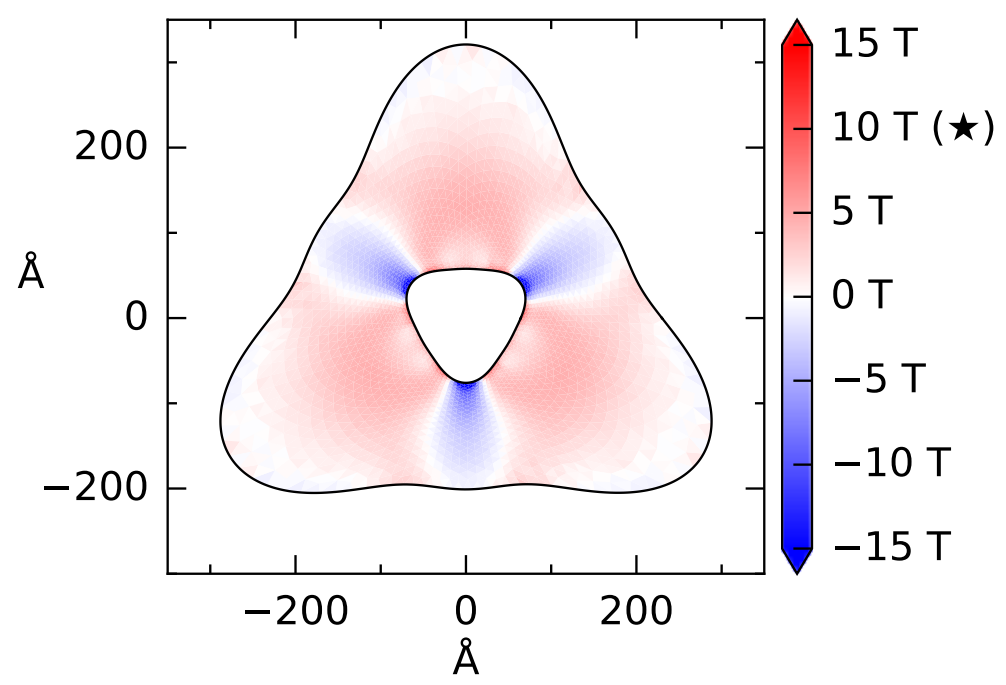

Figure S2: (color online) The PMF adopted by the graphene sheet when penalizing negative PMF values. The physical parameters were the same as those used in Fig. 2, and the target PMF was $10 \mathrm{~T}$ (indicated by $\star$ on the colorbar).

\section{Conductance of unpressurized devices}

The conductance of the unpressurized flower is approximately proportional to the inner contact radius; this is clearly observed in Figure S3a where the conductance for differently-sized flower devices is plotted. The devices were generated from the optimized flower shape shown in Fig. $2\left(R_{\text {in }} \approx 47 \AA\right.$ and $\left.200 \AA \leq R_{\text {out }} \leq 322 \AA\right)$, scaling inner and outer boundaries as $R_{\text {in }}^{\alpha}(\theta)=\alpha R_{\text {in }}(\theta)$ and $R_{\text {out }}^{\alpha}(\theta)=\alpha R_{\text {out }}(\theta)$. Larger $R_{\text {in }}^{\alpha}$ means a larger number of modes injected into the device; these modes are ballistically transmitted resulting in a higher conductance for larger $R_{\mathrm{in}}^{\alpha}$. Given that the device is a completely open system, resonances do not appear; however, Fabry-Perot oscillations with periodicity $\Delta E=\pi \hbar v_{F} / W_{\text {eff }}$ arise indicating the presence of an effective width $W_{\text {eff }}$ this quantity is the effective channel length of our device. ${ }^{8}$ For $\alpha=1$ we found $W_{\text {eff }}=223 \AA$ and $\Delta E \approx 0.03 t$; for $\alpha=2$ and $\alpha=3$ we extracted $\Delta E^{\alpha=2} \approx 0.014$ and $\Delta E^{\alpha=3} \approx 0.01$, indicating that $W_{\text {eff }}^{\alpha}=\alpha W_{\text {eff }}$ as anticipated. Since the geometry is unaltered when the device is scaled up, the value of the conductance at the Dirac point does not change, ${ }^{9}$ and $G(0)=2.4\left(2 e^{2} / h\right)$ for all values of $\alpha$. On the other hand, as observed in Corbino disks, ${ }^{9,10}$ the conductance near the Dirac point is energy independent in an interval that shrinks as $R_{\text {in }}^{\alpha}$ increases, which is related to the nearly-circular geometry 


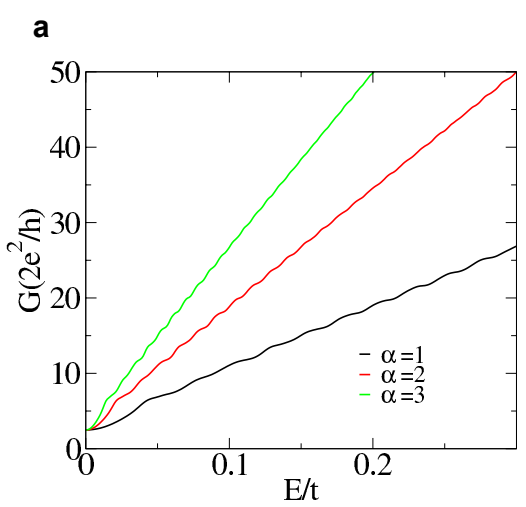

b

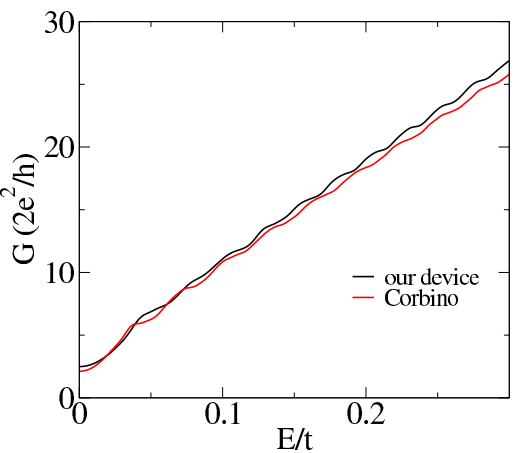

c

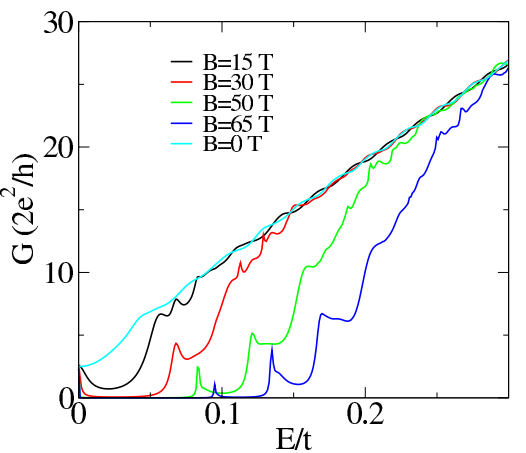

Figure S3: (color online) (a) Conductance of unpressurized devices scaled from the flower configuration (9)-(10) for scaling parameters $\alpha=1,2,3$. (b) Comparison of the conductance of the flower device and a Corbino disk with $R_{\text {in }}=47 \AA$ and $R_{\text {out }}=261 \AA$, both unpressurized. (c) Conductance of the unpressurized flower device for different values of real magnetic fields constant in magnitude and sign. Note how the conductance under a homogeneous field soon reaches zero at low energies, as one expects in a Corbino geometry where no edge states contribute to the current.

of the inner contact. Based on the effect that $R_{\text {in }}^{\alpha}$ has on the conductance of the device, we compared in Figure S3b the conductance of our $\alpha=1$ device that of an entirely circular Corbino disk of similar aspect ratio $R_{\text {in }} / R_{\text {out }}$ (specifically, $R_{\text {in }}=47 \AA$ and $R_{\text {out }}=261 \AA$ were chosen for the Corbino disk). The same behavior is observed in both devices, and their conductance very closely follow each other. This is explained by the fact that both are completely opened ballistic systems with circular or nearly-circular inner contact and similar inner/outer radius ratio. ${ }^{9,11}$

Switching on a (real for the moment) magnetic field $B$ introduces a new length scale in the problem. The resulting magneto-conductance can be understood by comparing the value of the cyclotron radius $r_{c}=\ell_{B}^{2} k_{F}\left(\right.$ where $\ell_{B}=\sqrt{\hbar / e B}$ is the magnetic length) with the variable distances between the inner and outer edges edges. ${ }^{10,12,13}$ For $r_{c}$ smaller than half the minimum edge-to-edge distance $\left(r_{c}<(200-47) / 2=76.5 \AA\right)$, electrons emitted from the inner contact cannot reach the outer one and resonant tunneling through Landau levels (LL) at energies $E_{n}=\left(\hbar v_{f} / \ell_{B}\right) \sqrt{2 n}$ emerges as the only transport mechanism. For example, in Figure S3c we see that at $B=50 \mathrm{~T}$ inner and outer edges are completely decoupled and 

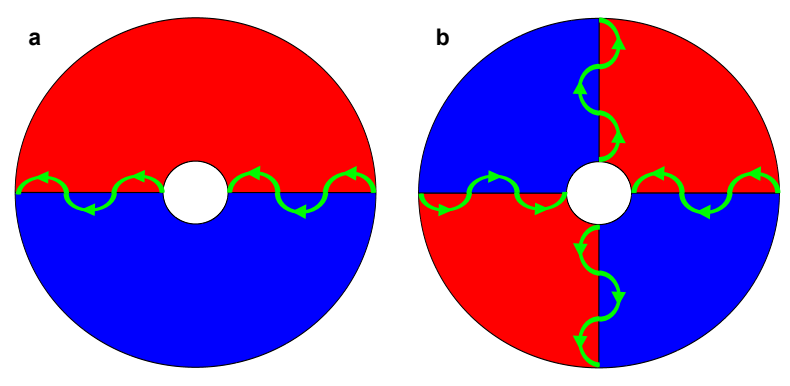

e

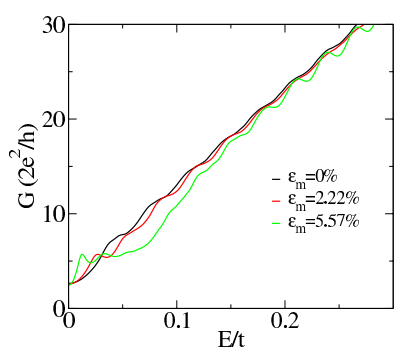

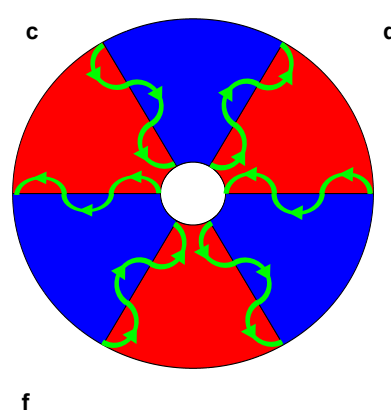
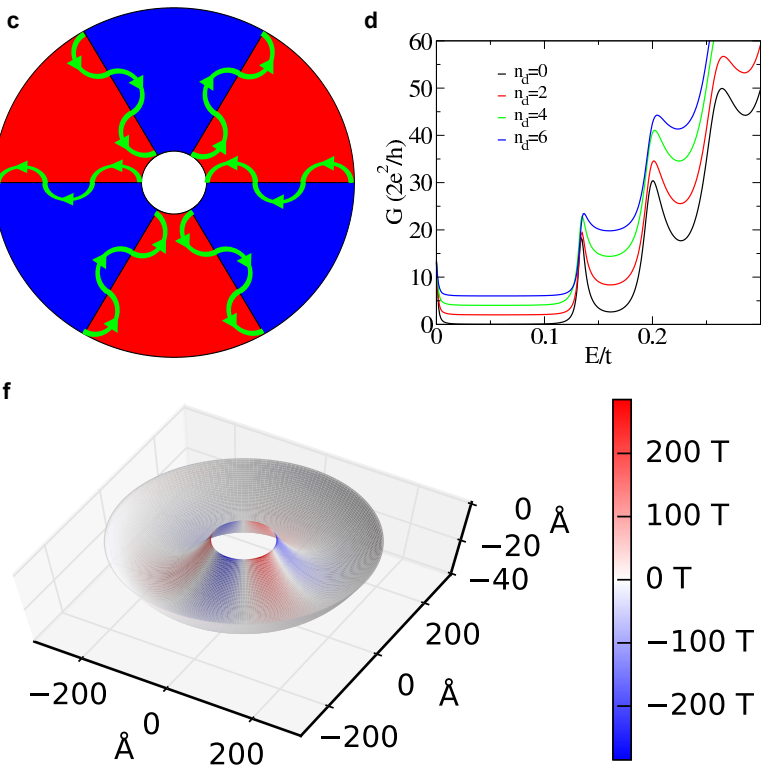

Figure S4: (color online) Schematic view of a Corbino disk and snake states in a real magnetic field with $n_{d}=2$ (a), $n_{d}=4$ (b), and $n_{d}=6$ (c) sectors of alternating polarity (sign); red represents positive and blue negative. (d) Conductance of a perfectly annular Corbino disk $\left(R_{\mathrm{in}}=280 \AA\right.$ and $\left.R_{\text {out }}=380 \AA\right)$ under those inhomogeneous magnetic fields. Note that, in contrast with the case shown in Figure S3c for a uniform external field, the conductance is now perfectly quantized, proportionally to the number of polarity change boundaries. (e) Conductance of an annular Corbino disk $\left(R_{\text {in }}=61 \AA\right.$ and $\left.R_{\text {out }}=261 \AA\right)$ under pressure (without any external magnetic field). (f) A surface plot (with exaggerated vertical scale) of an annulus (inner radius $61 \AA$, outer radius $261 \AA$ ) pressurized to 4 kbar, with a maximum vertical displacement of $20.8 \AA$, and a maximal strain of $5.76 \%$ (colors indicate PMF magnitude).

resonant tunneling through the $n=0 / 1 \mathrm{LL}$ is evident at the corresponding LL energies $E_{0}=0$ and $E_{1}=0.08 t\left(r_{c} \approx 53 \AA\right)$.

\section{Snake states in real inhomogeneous magnetic fields}

Previous studies of the transport properties of electrons under inhomogeneous magnetic fields have focused mostly on ribbon geometries. ${ }^{14-17}$ Here, we calculate the conductance of a graphene Corbino disk of $R_{\text {in }}=280 \AA$ and $R_{\text {out }}=380 \AA$ in an inhomogeneous real magnetic field to identify the similarities and differences in the conductance of ideal, sharp field boundaries arising from a real magnetic field in relation to PMF. Note that, whereas 
the spatial profiles of the real magnetic field discussed here are very unrealistic, those of the PMF discussed in the main text are, on the contrary, entirely realistic.

The field magnitude is set to $B_{0}$ over the entire annulus. The polarity or sign of $B_{0}$ alternates from positive to negative in neighboring sectors. This is schematically represented in Figures $\mathrm{S} 4 \mathrm{a}-\mathrm{c}$ for $n_{d}=2, n_{d}=4$ and $n_{d}=6$ sectors respectively, identifying where the polarity was defined as positive (red) and negative (blue). Based on that color code we highlighted the snake states as well as their direction. The number of snake states pointing outwards is $N_{s}=n_{d} / 2$, which has to be updated to $N_{s}=n_{d}$ when valley degeneracy is included. We set $B_{0}=125 \mathrm{~T}$ in order to perfectly observe $\mathrm{LL}$ in the case that the field is homogeneous $\left(n_{d}=0\right)$. Figure S4c shows the obtained conductance. The entirely homogeneous case $\left(n_{d}=0\right)$ exhibits the anticipated resonant peaks at the LL energies. Increasing the number of magnetic field sectors leads to conductance plateaus at $n_{d}\left(2 e^{2} / h\right)$ that are perfectly defined between the $n=1$ and $n=0$ LL.

\section{Conductance of optimized vs non-optimized Corbino de-}

\section{vices}

In order to illustrate that shape optimization is crucial to generate maximum confinement through PMF and to cause the emergence of snake states, we analyze here the transport characteristics of a non-optimized (strictly circular) Corbino device of equivalent dimensions, and under the same external pressure conditions. The pressurized Corbino disk of strictly circular geometry (radii $61 \AA$ and $261 \AA$ ) shown in Figure S4f has the conductance traces plotted in Figure S4e for different values of maximal strain $\varepsilon_{\mathrm{m}}$. Even though at $\varepsilon_{\mathrm{m}}=5.57 \%$ it displays a flattening of the conductance at low energies reminiscent of the behavior seen in the shape-optimized devices, this is explained by the much stronger PMF near the inner contact than within the annulus (see Figure S4f). These high PMF barriers on the inner contact force electrons to penetrate the device through regions where $B_{s} \approx 0$. But this 
confinement is limited to the inner contact region and does not extend to the whole annulus: for example, LL are not formed in the central regions of the device and this flattening doesn't develop into a full and flat step upon increasing the device dimensions, as happens in the shape-optimized cases shown in Fig. 4(c) of the main text. As a result, we contend that the flower geometry possesses superior properties for potential applications.

\section{Scaling up devices and their transport characteristics}

Of the many physical and geometric variables in the problem, only three are experimentally accessible. These are the device lengthscale $L$ (selected as $L_{\text {out }}$ from eq. (5) of the main text), the applied pressure $p$, and the target PMF $B_{0}$. In the main text we noted that these variables only affect the problem through the two dimensionless parameters $\bar{p}$ and $\kappa$ (provided in eq. (23) of this Supporting Information).

So, for instance, if one wished to find the applied pressure $p$ and target PMF $B_{0}$ for which a given lengthscale $L$ gave exactly the same flower shape through the optimization process, we would simply need to solve $\bar{p}=0.337$ and $\kappa=5.76 \times 10^{-5}$ for $p$ and $B_{0}$. In reality, however, this is too restrictive. Previously in this Supporting Information (Figure S1) we provided evidence that varying $\kappa$ has little effect on the optimal shape of the device. Indeed, the only effect of a larger $\kappa$ is that bending stiffness becomes a more important factor near the boundaries of the device. Since $\kappa=5.76 \times 10^{-5}$ is small enough to confine bending effects to the boundary, the selected flower shape will still be close to optimal for any value of $\kappa$ smaller than this value, i.e.

$$
\kappa \lesssim 5.76 \times 10^{-5}
$$

In the main text we show that the target PMF $\left(B_{0}\right)$ is not strictly attained over the whole device; a smaller and nearly constant effective PMF $\left(B_{\text {eff }}\right)$ appears instead. This effective field is responsible for the observed transport features, as we have established by determining $B_{\text {eff }}$ from the induced LL peaks in the LDOS or conductance traces. For our 
flower geometry, our transport and DOS calculations show that the field extracted from the induced LL spectrum $\left(B_{\text {eff }}\right)$ and $B_{0}$ are related by $B_{\text {eff }} \approx B_{0} / 2.2$.

Now, let us fix $\bar{p}=0.337$ in order to preserve the calculated flower shape. For a given lengthscale $L$ and applied pressure $p$, the formula

$$
\bar{p}=\frac{1}{C}\left(\frac{\hbar c}{a e}\right)^{3 / 2} \frac{p}{B_{0}^{3 / 2} L^{1 / 2}}=0.337
$$

tells us what target PMF $B_{0}$ would have outputted the flower shape as optimal. Conversely, replacing $B_{0}=2.2 B_{\text {eff }}$ in $(28)$, we can solve to find the effective PMF obtained by pressurizing the flower geometry of lengthscale $L$ with a given pressure $p$ :

$$
B_{\text {eff }}=\frac{1}{2.2 L^{1 / 3}}\left(\frac{\hbar c}{a e}\right)\left(\frac{p}{0.337 C}\right)^{2 / 3}
$$

Substituting the solution for $B_{0}$ from (28) into the restriction (27), using (23), gives a restriction on $p$ for a given lengthscale $L$ :

$$
p \gtrsim \frac{0.337}{L^{4} C^{1 / 2}}\left(\frac{D}{5.76 \times 10^{-5}}\right)^{3 / 2} .
$$

We wish to explore the parameter space $(L, p)$ for which snake states may be observed. First, electrons are to be injected from the inner contact with an energy $E \sim 0.5 E_{1}=$ $\frac{1}{2}\left(\frac{\hbar v_{F}}{\ell_{B}} \sqrt{2}\right)$. This guarantees that the energy of the electron is smaller than the energy of the first Landau level. We must thus ensure that Landau levels are formed in the bulk of the system, such that no transport occurs except through the radial interfaces where the PMF changes sign as snake-type states. Furthermore, in order to observe perfect quantization and perfect 1D-type transport, these modes must not be allowed to overlap.

Using a classical interpretation for the sake of obtaining specific estimates, these two criteria may be expressed as restrictions on the magnetic length $\ell_{B}$. Approximating the shape of the flower-shaped devices by an annulus of inner radius $\bar{R}_{\text {in }}$ and outer radius $\bar{R}_{\text {out }}$, the 


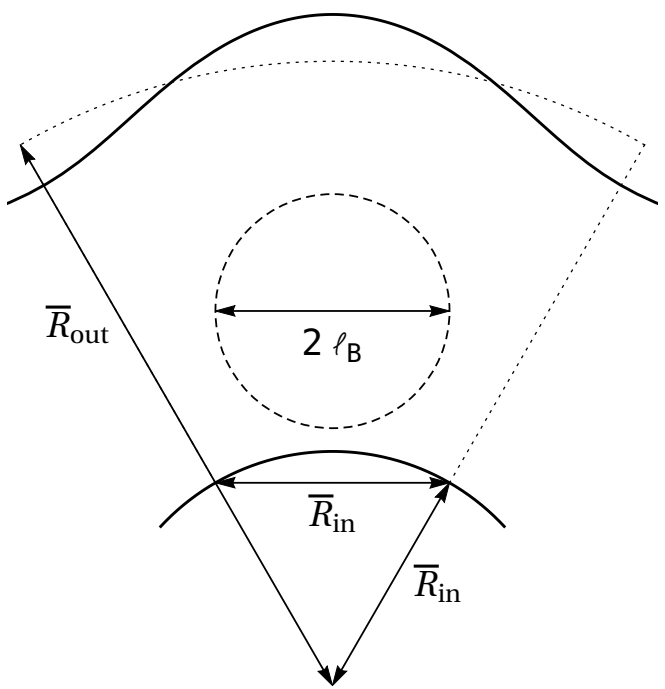

Figure S5: Schematic representation of a cyclotronic orbit in a petal (a $60^{\circ}$ sector) of the flower device. One must have $\ell_{B} \lesssim \bar{R}_{\text {in }} / 2$ in order to ensure that Landau levels are well developed within the inside regions of each petal, while simultaneously avoiding overlap between snake states propagating along neighboring radial paths.

diameter of the cyclotron orbit $2 \ell_{B}$ must be shorter than both the annulus width $\bar{R}_{\text {out }}-\bar{R}_{\text {in }}$ and the inner width of a single $\pi / 3$-sector (approximately $\bar{R}_{\text {in }}$ ). See Figure S5 for a graphical interpretation. Overbars here signify the mean radius of the shape.

For the dimensions of the optimal shape reported in the main text, $\bar{R}_{\text {in }} \sim 47 \AA$ and $\bar{R}_{\text {out }} \sim 245 \AA \sim 5 \bar{R}_{\text {in }}$. Thus, for this particular shape, the restrictions on $\ell_{B}$ become

$$
\begin{gathered}
\ell_{B}<\frac{1}{2} \bar{R}_{\text {in }} \sim \frac{\bar{R}_{\text {out }}}{10}, \\
\ell_{B}<\frac{1}{2}\left(\bar{R}_{\text {out }}-\bar{R}_{\text {in }}\right) \sim \frac{4 \bar{R}_{\text {out }}}{5} .
\end{gathered}
$$

The first of these is the more restrictive condition, and we combine this with (29) and $\ell_{B}=\sqrt{\hbar /\left(e B_{\text {eff }}\right)}$ to give

$$
p \gtrsim \frac{0.337 C L^{1 / 2}}{\left(\bar{R}_{\text {out }} / 10\right)^{3}}\left(\frac{2.2 a}{c}\right)^{3 / 2} .
$$

Since the preceding calculation uses a classical interpretation and geometry, it can only be considered as an order-of-magnitude estimate for the true limit as our particular problem is not in the classical regime. In order to more precisely fix this constraint, we take into 
account the observation in Fig. 4a (main text) that the device with $\varepsilon_{\mathrm{m}}=6.11 \%$ is at the threshold of perfect conductance quantization and strictly 1D transport (because the plateau is nearly perfectly developed at this pressurization already). We will demonstrate that, if the constraint (31) is relaxed slightly to $\ell_{B}<L / 8$ (as $\left.\bar{R}_{\text {out }} \sim L\right)$, that particular device falls precisely on the threshold line and we, accordingly, use this latter condition to identify the range of parameters that we expect should lead to the same qualitative PMF and transport behavior in scaled devices. Under this assumption the constraint (33) becomes

$$
p \gtrsim \frac{8^{3} \times 0.337 C}{L^{5 / 2}}\left(\frac{2.2 a}{c}\right)^{3 / 2}
$$

(a)

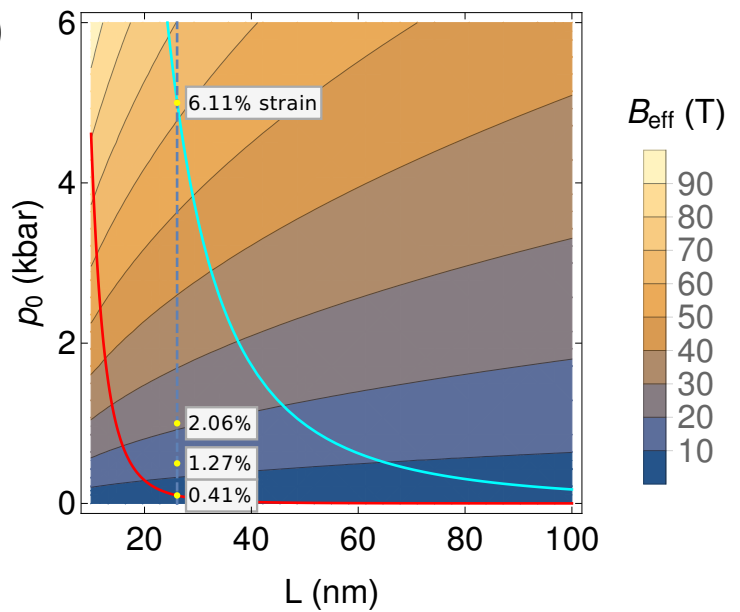

(b)

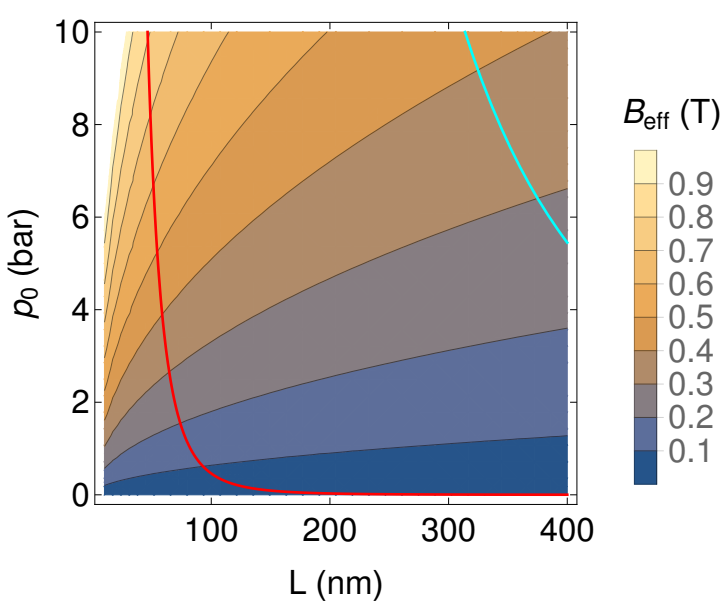

Figure S6: (a) A contour plot of the effective PMF $B_{\text {eff }}$ for a range of small lengthscales $L$ and applied pressures $p$ up to $6 \mathrm{kbar}$. The constraints (30) and (34) on $p$ are superimposed as red and cyan curves respectively; these constraints are satisfied in the region of parameters above each line. The four highlighted configurations correspond to the configuration optimized for $\varepsilon_{\mathrm{m}}=0.41 \%$ and the three strained configurations investigated in Fig. 4(a)-(b) of the main text, all at $L=L_{\text {out }}=26.1 \mathrm{~nm}$. (b) As plot (a), but for a larger (and more experimentally realizable) range of lengthscales $L$ (note the different units of the vertical axes).

In Figure S6(a) we plot the effective PMF from eq. (29) for a range of small devices (lengthscales up to $100 \mathrm{~nm}$ ). On this we superimpose the two curves corresponding to the restrictions on $p$, from relations (30) and (34). Values of $p$ above the former (red) curve have small enough bending stiffness effects that the originally calculated device will still have the optimal shape; this constraint is easily satisfied for essentially all experimentally 


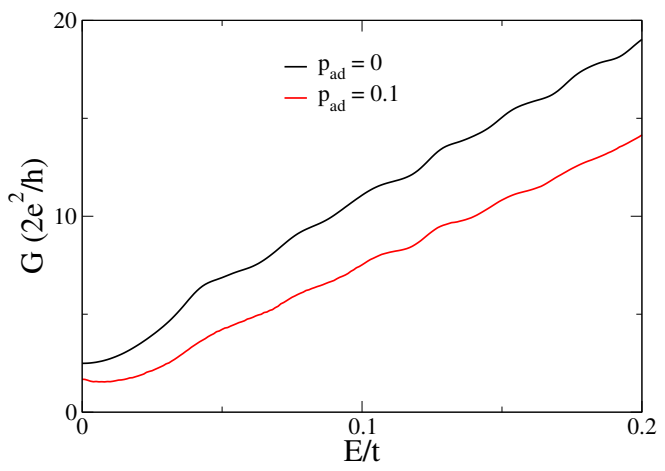

Figure S7: Conductance of the unpressurized device with and without adatoms.

relevant lengthscales and pressures. Values of $p$ above the cyan curve are needed in order to observe strictly one-dimensional transport. Note that the $\varepsilon_{\mathrm{m}}=6.11 \%$ solution lies directly on the cyan curve, confirming our previous statement regarding the transport characteristics of this device reported in Fig. 4a of the main text. In Figure S6(b) we have plotted the same results but for a larger range of lengthscales (up to $400 \mathrm{~nm}$ ). A device of lengthscale $L=400 \mathrm{~nm}$ has an inner radius of $78 \mathrm{~nm}$, resulting in a pattern which is perfectly feasible with standard lithography. At this lengthscale, the minimum pressure for the observation of onedimensional transport is found at the intersection with the cyan curve, i.e. at $p=5.45$ bar. This results in an effective PMF of $0.26 \mathrm{~T}$ and (by design) a magnetic length of $\ell_{B}=50 \mathrm{~nm}$.

Finally, note that, as follows from this discussion, it is not the magnitude of the generated PMF alone that determines whether the $1 \mathrm{D}$ transport regime is realized or not. It is rather the combination of the field magnitude, through its influence in the magnetic length and "orbit" size, with the characteristic device dimensions that determines the favorable conditions for the development of robust LL in the bulk of the device, and decoupled snake-type states along the radial interfaces.

\section{Disorder in the unpressurized flower device}

In Figure S7 we show the conductance of the $\alpha=1$ unpressurized flower device in the presence of adatoms, the adatoms are distributed over the whole area of the device. We can see that the conductance is reduced by $\sim 60 \%$ for $E_{F}=0.04 t$, . For larger devices this value 
should be increased given that the number of angular momentum channels $j_{m} \propto R_{\text {in }}$ and the conductance is proportional to $j_{m}$.

\section{References}

(1) Jones, G. W.; Pereira, V. M. New J. Phys. 2014, 16, 093044.

(2) Ciarlet, P. G. In The Finite Element Method for Elliptic Problems, 1st ed.; Lions, J. L., Papanicolau, G., Rockafellar, R. T., Eds.; Studies in Mathematics and its Applications; North-Holland: Amsterdam, 1978; Vol. 4.

(3) Zienkiewicz, O. C.; Zhu, J. Z. Int. J. Numer. Meth. Eng. 1992, 33, 1331-1364.

(4) Kudin, K. N.; Scuseria, G. E.; Yakobson, B. I. Phys. Rev. B 2001, 64, 235406.

(5) Wei, X.; Fragneaud, B.; Marianetti, C. A.; Kysar, J. W. Phys. Rev. B 2009, 80, 205407.

(6) Zhou, J.; Huang, R. J. Mech. Phys. Solids 2008, 56, 1609.

(7) Midtvedt, D.; Lewenkopf, C. H.; Croy, A. $2 D$ Mater. 2016, 3, 011005.

(8) Ng, K. K.; Brews, J. R. IEEE Circuits and Devices Magazine 1990, 6, 33-38.

(9) Rycerz, A.; Recher, P.; Wimmer, M. Phys. Rev. B 2009, 80, 125417.

(10) Bahamon, D. A.; Castro Neto, A. H.; Pereira, V. M. Phys. Rev. B 2013, 88, 235433.

(11) Rut, G.; Rycerz, A. Phys. Rev. B 2016, 93, 075419.

(12) Kirczenow, G. J Phys.: Cond. Mat. 1994, 6, L583.

(13) Prada, E.; San-Jose, P.; Wunsch, B.; Guinea, F. Phys. Rev. B 2007, 75, 113407.

(14) Müller, J. E. Phys. Rev. Lett. 1992, 68, 385-388.

(15) Gu, B.-Y.; Sheng, W.-D.; Wang, X.-H.; Wang, J. Phys. Rev. B 1997, 56, 13434-13441. 
(16) Oroszlány, L.; Rakyta, P.; Kormányos, A.; Lambert, C. J.; Cserti, J. Phys. Rev. B 2008, 77, 081403.

(17) Ghosh, T. K.; De Martino, A.; Häusler, W.; Dell'Anna, L.; Egger, R. Phys. Rev. B 2008, 77, 081404. 